\title{
Rational Computations of the Topological $K$-Theory of Classifying Spaces of Discrete Groups
}

\author{
Wolfgang Lück* \\ Fachbereich Mathematik \\ Universität Münster \\ Einsteinstr. 62 \\ 48149 Münster \\ Germany
}

February 17, 2019

\begin{abstract}
We compute rationally the topological (complex) K-theory of the classifying space $B G$ of a discrete group provided that $G$ has a cocompact $G$ - $C W$-model for its classifying space for proper $G$-actions. For instance word-hyperbolic groups and cocompact discrete subgroups of connected Lie groups satisfy this assumption. The answer is given in terms of the group cohomology of $G$ and of the centralizers of finite cyclic subgroups of prime power order. We also analyze the multiplicative structure.
\end{abstract}

Key words: topological $K$-theory, classifying spaces of groups.

Mathematics Subject Classification 2000: 55N15.

\section{Introduction and Statements of Results}

The main result of this paper is:

Theorem 0.1 (Main result). Let $G$ be a discrete group. Denote by $K^{*}(B G)$ the topological (complex) K-theory of its classifying space BG. Suppose that there is a cocompact $G-C W$-model for the classifying space $\underline{E} G$ for proper $G$ actions.

*email: lueck@math.uni-muenster.de

www: http://www.math.uni-muenster.de/u/lueck/

FAX: 492518338370 
Then there is a $\mathbb{Q}$-isomorphism

$$
\begin{aligned}
\overline{\operatorname{ch}}_{G}^{n}: K^{n}(B G) \otimes_{\mathbb{Z}} \mathbb{Q} \stackrel{\cong}{\rightrightarrows} & \\
& \left(\prod_{i \in \mathbb{Z}} H^{2 i+n}(B G ; \mathbb{Q})\right) \times \prod_{p \text { prime }} \prod_{(g) \in \operatorname{con}_{p}(G)}\left(\prod_{i \in \mathbb{Z}} H^{2 i+n}\left(B C_{G}\langle g\rangle ; \mathbb{Q}_{p}\right)\right),
\end{aligned}
$$

where $\operatorname{con}_{p}(G)$ is the set of conjugacy classes ( $g$ ) of elements $g \in G$ of order $p^{d}$ for some integer $d \geq 1$ and $C_{G}\langle g\rangle$ is the centralizer of the cyclic subgroup $\langle g\rangle$ generated by $g$.

The classifying space $\underline{E} G$ for proper $G$-actions is a proper $G$-CW-complex such that the $H$-fixed point set is contractible for every finite subgroup $H \subseteq G$. It has the universal property that for every proper $G$ - $C W$-complex $X$ there is up to $G$-homotopy precisely one $G$-map $f: X \rightarrow \underline{E} G$. Recall that a $G-C W$ complex is proper if and only if all its isotropy groups are finite, and is finite if and only if it is cocompact. The assumption in Theorem 0.1 that there is a cocompact $G-C W$-model for the classifying space $\underline{E} G$ for proper $G$-actions is satisfied for instance if $G$ is word-hyperbolic in the sense of Gromov, if $G$ is a cocompact subgroup of a Lie group with finitely many path components, if $G$ is a finitely generated one-relator group, if $G$ is an arithmetic group, a mapping class group of a compact surface or the group of outer automorphisms of a finitely generated free group. For more information about $\underline{E} G$ we refer for instance to [8] and [21]. We will prove Theorem 0.1 in Section 4.

We will also investigate the multiplicative structure on $K^{n}(B G) \otimes_{\mathbb{Z}} \mathbb{Q}$ in Section 5. If one is willing to complexify, one can show:

Theorem 0.2 (Multiplicative structure). Let $G$ be a discrete group. Suppose that there is a cocompact $G-C W$-model for the classifying space $\underline{E} G$ for proper $G$-actions.

Then there is a $\mathbb{C}$-isomorphism

$$
\begin{aligned}
& \overline{\operatorname{ch}}_{G, \mathbb{C}}^{n}: K^{n}(B G) \otimes_{\mathbb{Z}} \mathbb{C} \stackrel{\cong}{\rightrightarrows} \\
& \left(\prod_{i \in \mathbb{Z}} H^{2 i+n}(B G ; \mathbb{C})\right) \times \prod_{p} \prod_{\text {prime }}\left(\prod_{(g) \in \operatorname{con}_{p}(G)}\left(\prod_{i \in \mathbb{Z}} H^{2 i+n}\left(B C_{G}\langle g\rangle ; \mathbb{Q}_{p}^{\widehat{p}} \otimes_{\mathbb{Q}} \mathbb{C}\right)\right),\right.
\end{aligned}
$$

which is compatible with the standard multiplicative structure on $K^{*}(B G)$ and the one on the target given by

$$
\left(a, u_{p,(g)}\right) \cdot\left(b, v_{p,(g)}\right)=\left(a \cdot b,\left(a \cdot v_{p,(g)}+b \cdot u_{p,(g)}+u_{p,(g)} \cdot v_{p,(g)}\right)\right)
$$

for

$$
\begin{aligned}
(g) & \in \operatorname{con}_{p}(G) ; \\
a, b & \in \prod_{i \in \mathbb{Z}} H^{2 i+*}(B G ; \mathbb{C}) ; \\
u_{p,(g)}, v_{p,(g)} & \in \prod_{i \in \mathbb{Z}} H^{2 i+*}\left(B C_{G}\langle g\rangle ; \mathbb{Q}_{p}^{-} \otimes_{\mathbb{Q}} \mathbb{C}\right),
\end{aligned}
$$


and the structures of a graded commutative ring on $\prod_{i \in \mathbb{Z}} H^{2 i+*}(B G ; \mathbb{C})$ and $\prod_{i \in \mathbb{Z}} H^{2 i+*}\left(B C_{G}\langle g\rangle ; \widehat{\mathbb{Q}_{p}} \otimes_{\mathbb{Q}} \mathbb{C}\right)$ coming from the cup-product and the obvious $\prod_{i \in \mathbb{Z}} H^{2 i+*}(B G ; \mathbb{C})$-module structure on $\prod_{i \in \mathbb{Z}} H^{2 i+*}\left(B C_{G}\langle g\rangle ; \mathbb{Q}_{p} \otimes_{\mathbb{Q}} \mathbb{C}\right)$ coming from the canonical maps $B C_{G}\langle g\rangle \rightarrow B G$ and $\mathbb{C} \rightarrow \widehat{\mathbb{Q}_{p}} \otimes_{\mathbb{Q}} \mathbb{C}$.

In Section 6 we will prove Theorem 0.1 and Theorem 0.2 under weaker finiteness assumptions than stated above.

If $G$ is finite, we get the following integral computation of $K^{*}(B G)$. Throughout the paper $R(G)$ will be the complex representation ring and $\mathbb{I}_{G}$ be its augmentation ideal, i.e. the kernel of the ring homomorphism $R(G) \rightarrow \mathbb{Z}$ sending $[V]$ to $\operatorname{dim}_{\mathbb{C}}(V)$. If $G_{p} \subseteq G$ is a $p$-Sylow subgroup, restriction defines a map $\mathbb{I}(G) \rightarrow \mathbb{I}\left(G_{p}\right)$. Let $\mathbb{I}_{p}(G)$ be the quotient of $\mathbb{I}(G)$ by the kernel of this map. This is independent of the choice of the $p$-Sylow subgroup since two $p$ Sylow subgroups of $G$ are conjugate. There is an obvious isomorphism from $\mathbb{I}_{p}(G) \stackrel{\cong}{\rightarrow} \operatorname{im}\left(\mathbb{I}(G) \rightarrow \mathbb{I}\left(G_{p}\right)\right)$. We will prove in Section 3

Theorem 0.3. (K-theory of $B G$ for finite groups $G$ ). Let $G$ be a finite group. For a prime $p$ denote by $r(p)=\left|\operatorname{con}_{p}(G)\right|$ the number of conjugacy classes $(g)$ of elements $g \in G$ whose order $|g|$ is $p^{d}$ for some integer $d \geq 1$. Then there are isomorphisms of abelian groups

$$
\begin{aligned}
K^{0}(B G) \cong \mathbb{Z} \times \prod_{\text {prime }} \mathbb{I}_{p}(G) \otimes_{\mathbb{Z}} \mathbb{Z}_{p} \cong \mathbb{Z} \times \prod_{\text {p prime }}\left(\mathbb{Z}_{p}\right)^{r(p)} \\
K^{1}(B G) \cong 0
\end{aligned}
$$

The isomorphism $K^{0}(B G) \stackrel{\cong}{\longrightarrow} \mathbb{Z} \times \prod_{p \text { prime }} \mathbb{I}_{p}(G) \otimes_{\mathbb{Z}} \mathbb{Z}_{p}$ is compatible with the standard ring structure on the source and the ring structure on the target given by

$$
\left(m, u_{p} \otimes a_{p}\right) \cdot\left(n, v_{p} \otimes b_{p}\right)=\left(m n,\left(m v_{p} \otimes b_{p}+n u_{p} \otimes a_{p}+\left(u_{p} v_{p}\right) \otimes\left(a_{p} b_{p}\right)\right)\right.
$$

for $m, n \in \mathbb{Z}, u_{p}, v_{p} \in \mathbb{I}_{p}(G)$ and $a_{p}, b_{p} \in \mathbb{Z}_{p}$ and the obvious multiplication in $\mathbb{Z}, \mathbb{I}_{p}(G)$ and $\mathbb{Z}_{p}$.

The additive version of Theorem 0.3 has already been explained in [15, page 125]. We will and need to show a stronger statement about the pro-group $\left\{\mathbb{I}_{G} /\left(\mathbb{I}_{G}\right)^{n+1}\right\}$ in Theorem $3.5(\mathrm{~b})$.

A version of Theorem 0.1 for topological $K$-theory with coefficients in the $p$-adic integers has been proved by Adem [1], [2] using the Atiyah-Segal completion theorem for the finite group $G / G^{\prime}$ provided that $G$ contains a torsionfree subgroup $G^{\prime}$ of finite index. Our methods allow to drop this condition, to deal with $K^{*}(B G) \otimes_{\mathbb{Z}} \mathbb{Q}$ directly and study systematically the multiplicative structure for $K^{*}(B G) \otimes_{\mathbb{Z}} \mathbb{C}$. They are based on the equivariant cohomological Chern character of $[20]$.

For integral computations of the $K$-theory and $K$-homology of classifying spaces of groups we refer to [16].

The paper is organized as follows: 
1. Borel Cohomology and Rationalization

2. Some Preliminaries about Pro-Modules

3. The K-Theory of the Classifying Space of a Finite Group

4. Proof of the Main Result

5. Multiplicative Structures

6. Weakening the Finiteness Conditions

7. Examples and Further Remarks

References

The author wants to the thank the Max Planck Institute for Mathematics in Bonn for its hospitality during his stay from April 2005 until July 2005 when this paper was written.

\section{Borel Cohomology and Rationalization}

Denote by GROUPOIDS the category of small groupoids. Let $\Omega$-SPECTRA be the category of $\Omega$-spectra, where a morphism $\mathbf{f}: \mathbf{E} \rightarrow \mathbf{F}$ is a sequence of maps $f_{n}: E_{n} \rightarrow F_{n}$ compatible with the structure maps and we work in the category of compactly generated spaces (see for instance [11, Section 1]). A contravariant GROUPOIDS- $\Omega$-spectrum is a contravariant functor $\mathbf{E}$ : GROUPOIDS $\rightarrow$ $\Omega$-SPECTRA.

Let $\mathbf{E}$ be a (non-equivariant) $\Omega$-spectrum. We can associate to it a contravariant GROUPOIDS- $\Omega$-spectrum

$$
\mathbf{E}_{\text {Bor }}: \text { GROUPOIDS } \rightarrow \Omega \text {-SPECTRA; } \mathcal{G} \mapsto \operatorname{map}(B \mathcal{G} ; \mathbf{E}),
$$

where $B \mathcal{G}$ is the classifying space associated to $\mathcal{G}$ and $\operatorname{map}(B \mathcal{G} ; \mathbf{E})$ is the obvious mapping space spectrum (see for instance [11, page 208 and Definition 3.10 on page 224]). In the sequel we use the notion of an equivariant cohomology theory $\mathcal{H}_{\text {? }}^{*}$ with values in $R$-modules of [20, Section 1]. It assigns to each (discrete) group $G$ a $G$-cohomology theory $\mathcal{H}_{G}^{*}$ with values in the category of $R$-modules on the category of pairs of $G-C W$-complexes, where $*$ runs through $\mathbb{Z}$. Let $H_{?}^{*}\left(-, \mathbf{E}_{\mathrm{Bor}}\right)$ be the to $\mathbf{E}_{\mathrm{Bor}}$ associated equivariant cohomology theory with values in $\mathbb{Z}$-modules satisfying the disjoint union axiom, which has been constructed in [20, Example 1.8]. For a given discrete group $G$ and a $G-C W$-pair $(X, A)$ and $n \in \mathbb{Z}$ we get a natural identification

$$
H_{G}^{n}\left(X, A ; \mathbf{E}_{\mathrm{Bor}}\right)=H^{n}\left(E G \times_{G}(X, A) ; \mathbf{E}\right),
$$

where $H^{*}(-; \mathbf{E})$ is the (non-equivariant) cohomology theory associated to $\mathbf{E}$. It is induced by the following composite of equivalences of $\Omega$-spectra

$$
\begin{aligned}
& \operatorname{map}_{\mathrm{Or}(G)}\left(\operatorname{map}_{G}(G / ?, X)^{G}, \operatorname{map}\left(B \mathcal{G}^{G}(G / H), \mathbf{E}\right)\right) \\
& \rightarrow \operatorname{map}_{\mathrm{Or}(G)}\left(\operatorname{map}_{G}(G / ?, X)^{G}, \operatorname{map}\left(E G \times_{G} G / ?, \mathbf{E}\right)\right) \\
& \rightarrow \operatorname{map}\left(\operatorname{map}_{G}(G / ?, X)^{G} \otimes_{\mathrm{Or}(G)} E G \times_{G} G / ?, \mathbf{E}\right) \rightarrow \operatorname{map}\left(E G \times_{G} X, \mathbf{E}\right)
\end{aligned}
$$


using the notation of [20]. In the literature $H^{n}\left(E G \times_{G}(X, A) ; \mathbf{E}\right)$ is called the equivariant Borel cohomology of $(X, A)$ with respect to the (non-equivariant) cohomology theory $H^{*}(-; \mathbf{E})$.

Our main example for $\mathbf{E}$ will be the topological $K$-theory spectrum $\mathbf{K}$, whose associated (non-equivariant) cohomology theory $H^{*}(-; \mathbf{K})$ is topological $K$-theory $K^{*}$.

There is a functor

$$
\text { Rat: } \Omega \text {-SPECTRA } \rightarrow \Omega \text {-SPECTRA }, \quad \mathbf{E} \mapsto \boldsymbol{\operatorname { R a t }}(\mathbf{E})=\mathbf{E}_{(0)},
$$

which assigns to an $\Omega$-spectrum $\mathbf{E}$ its rationalization $\mathbf{E}_{(0)}$. The homotopy groups $\pi_{k}\left(\mathbf{E}_{(0)}\right)$ come with a canonical structure of a $\mathbb{Q}$-module. There is a natural transformation

$$
\mathbf{i}(\mathbf{E}): \mathbf{E} \rightarrow \mathbf{E}_{(0)}
$$

which induces isomorphisms

$$
\pi_{k}(\mathbf{i}(\mathbf{E})): \pi_{k}(\mathbf{E}) \otimes_{\mathbb{Z}} \mathbb{Q} \stackrel{\cong}{\rightrightarrows} \pi_{k}\left(\mathbf{E}_{(0)}\right) .
$$

Composing $\mathbf{E}_{\text {Bor }}$ with Rat yields a contravariant $\operatorname{Or}(G)-\Omega$-spectrum denoted by $\left(\mathbf{E}_{\mathrm{Bor}}\right)_{(0)}$. We obtain an equivariant cohomology theory with values in $\mathbb{Q}$ modules by $H_{?}^{*}\left(-;\left(\mathbf{E}_{\mathrm{Bor}}\right)_{(0)}\right)$. The map $\mathbf{i}$ induces a natural transformation of equivariant cohomology theories

$$
i_{?}^{*}(-; \mathbf{E}): H_{?}^{*}\left(-; \mathbf{E}_{\mathrm{Bor}}\right) \otimes_{\mathbb{Z}} \mathbb{Q} \rightarrow H_{?}^{*}\left(-;\left(\mathbf{E}_{\mathrm{Bor}}\right)_{(0)}\right) .
$$

Lemma 1.6. If $G$ is a group $G$ and $(X, A)$ is a relative finite $G-C W$-pair, then

$$
i_{G}^{n}(X, A ; \mathbf{E}): H_{G}^{n}\left(X, A ; \mathbf{E}_{\mathrm{Bor}}\right) \otimes_{\mathbb{Z}} \mathbb{Q} \rightarrow H_{G}^{n}\left(X, A ;\left(\mathbf{E}_{\mathrm{Bor}}\right)_{(0)}\right)
$$

is a $\mathbb{Q}$-isomorphism for all $n \in \mathbb{Z}$.

Proof. The transformation $i_{G}^{*}(-; \mathbf{E})$ is a natural transformation of $G$-cohomology theories since $\mathbb{Q}$ is flat over $\mathbb{Z}$. One easily checks that it induces a bijection in the case $X=G / H$, since then there is a commutative square with obvious isomorphisms as vertical maps and the isomorphism of (1.4) as lower horizontal arrow

$$
\begin{array}{ccc}
H_{G}^{k}\left(G / H ; \mathbf{E}_{\mathrm{Bor}}\right) \otimes_{\mathbb{Z}} \mathbb{Q} & \stackrel{i_{G}^{*}(G / H ; \mathbf{E})}{\longrightarrow} & H_{G}^{k}\left(G / H ;\left(\mathbf{E}_{\mathrm{Bor}}\right)_{(0)}\right) \\
\cong & \downarrow \\
\pi_{-k}(\operatorname{map}(B H, \mathbf{E})) \otimes_{\mathbb{Z}} \mathbb{Q} & \stackrel{\downarrow}{\stackrel{\pi_{-k}(\mathbf{i}(\operatorname{map}(B H, \mathbf{E})))}{\longrightarrow}} \pi_{-k}\left((\operatorname{map}(B H, \mathbf{E}))_{(0)}\right)
\end{array}
$$

By induction over the number of $G$-cells using Mayer-Vietoris sequences one shows that $i_{G}^{*}(X, A)$ is an isomorphism for all relative finite $G$ - $C W$-pairs $(X, A)$. 
Remark 1.7. (Comparison of the various rationalizations). Notice that $i_{G}^{*}(X, A, \mathbf{E})$ of $(1.5)$ is not an isomorphism for all $G$ - $C W$-pairs $(X, A)$ because the source does not satisfy the disjoint union axiom for arbitrary index sets in contrast to the target. The point is that $-\otimes_{\mathbb{Z}} \mathbb{Q}$ is compatible with direct sums but not with direct products.

Since $H_{?}^{*}\left(-;\left(\mathbf{K}_{\mathrm{Bor}}\right)_{(0)}\right)$ is an equivariant cohomology theory with values in $\mathbb{Q}$-modules satisfying the disjoint union axiom, we can use the equivariant cohomological Chern character of $[20]$ to compute $H_{G}^{*}\left(\underline{E} G ;\left(\mathbf{K}_{\mathrm{Bor}}\right)_{(0)}\right)$ for all groups $G$.

This is also true for the equivariant cohomology theory with values in $\mathbb{Q}$ modules satisfying the disjoint union axiom $H_{?}^{*}\left(-;\left(\mathbf{K}_{(0)}\right)_{\text {Bor }}\right)$. (Here we have changed the order of Bor and (0).) But this a much worse approximation of $K^{k}(B G) \otimes_{\mathbb{Z}} \mathbb{Q}$ than $H_{G}^{*}\left(B G ;\left(\mathbf{E}_{\text {Bor }}\right)_{(0)}\right)$. Namely, i induces using the universal property of Rat a natural map of contravariant GROUPOIDS- $\Omega$-spectra

$$
\left(\mathbf{K}_{\text {Bor }}\right)_{(0)} \rightarrow\left(\mathbf{K}_{(0)}\right)_{\text {Bor }}
$$

and thus a natural map

$$
H_{G}^{k}\left(X ;\left(\mathbf{K}_{\mathrm{Bor}}\right)_{(0)}\right) \rightarrow H_{G}^{k}\left(X ;\left(\mathbf{K}_{(0)}\right)_{\text {Bor }}\right)
$$

but this map is in general not an isomorphism. Namely, it is not bijective for $X=G / H$ for finite non-trivial $H$ and $k=0$. In this case the source turns out to be

$$
\pi_{0}\left((\operatorname{map}(B H ; \mathbf{K}))_{(0)}\right) \cong K^{0}(B H) \otimes_{\mathbb{Z}} \mathbb{Q} \cong \mathbb{Q} \times \prod_{p|| H \mid}\left(\mathbb{Q}_{p}^{-}\right)^{r(p)}
$$

for $r(p)$ the number of conjugacy classes $(h)$ of non-trivial elements $h \in H$ of $p$ power order, and the target is $K^{0}(B H ; \mathbb{Q})$ which turns out to be isomorphic to $\mathbb{Q}$ since the rational cohomology of $B H$ agrees with the one of the one-point-space.

As mentioned before we want to use the equivariant cohomological Chern character of $[20]$ to compute $H_{G}^{*}\left(X ;\left(\mathbf{K}_{\mathrm{Bor}}\right)_{(0)}\right)$. This requires a careful analysis of the contravariant functor

$$
\text { FGINJ } \rightarrow \mathbb{Q}-\text { MOD }, \quad H \mapsto H_{G}^{k}\left(G / H ;\left(\mathbf{K}_{\text {Bor }}\right)_{(0)}\right)=K^{k}(B H) \otimes_{\mathbb{Z}} \mathbb{Q},
$$

from the category FGINJ of finite groups with injective group homomorphisms as morphisms to the category $\mathbb{Q}$ - MOD of $\mathbb{Q}$-modules. It will be carried out in Section 3 after some preliminaries in Section 2.

\section{Some Preliminaries about Pro-Modules}

It will be crucial to handle pro-systems and pro-isomorphisms and not to pass directly to inverse limits. In this section we fix our notation for handling 
pro- $R$-modules for a commutative ring $R$, where ring always means associative ring with unit. For the definitions in full generality see for instance [3, Appendix] or $[6, \S 2]$.

For simplicity, all pro- $R$-modules dealt with here will be indexed by the positive integers. We write $\left\{M_{n}, \alpha_{n}\right\}$ or briefly $\left\{M_{n}\right\}$ for the inverse system

$$
M_{0} \stackrel{\alpha_{1}}{\longleftarrow} M_{1} \stackrel{\alpha_{2}}{\longleftarrow} M_{2} \stackrel{\alpha_{3}}{\longleftarrow} M_{3} \stackrel{\alpha_{4}}{\longleftarrow} \ldots
$$

and also write $\alpha_{n}^{m}:=\alpha_{m+1} \circ \cdots \circ \alpha_{n}: G_{n} \rightarrow G_{m}$ for $n>m$ and put $\alpha_{n}^{n}=\operatorname{id}_{G_{n}}$. For the purposes here, it will suffice (and greatly simplify the notation) to work with "strict" pro-homomorphisms $\left\{f_{n}\right\}:\left\{M_{n}, \alpha_{n}\right\} \rightarrow\left\{N_{n}, \beta_{n}\right\}$, i.e. a collection of homomorphisms $f_{n}: M_{n} \rightarrow N_{n}$ for $n \geq 1$ such that $\beta_{n} \circ f_{n}=f_{n-1} \circ \alpha_{n}$ holds for each $n \geq 2$. Kernels and cokernels of strict homomorphisms are defined in the obvious way.

A pro- $R$-module $\left\{M_{n}, \alpha_{n}\right\}$ will be called pro-trivial if for each $m \geq 1$, there is some $n \geq m$ such that $\alpha_{n}^{m}=0$. A strict homomorphism $f:\left\{M_{n}, \alpha_{n}\right\} \rightarrow$ $\left\{N_{n}, \beta_{n}\right\}$ is a pro-isomorphism if and only if $\operatorname{ker}(f)$ and $\operatorname{coker}(f)$ are both protrivial, or, equivalently, for each $m \geq 1$ there is some $n \geq m$ such that $\operatorname{im}\left(\beta_{n}^{m}\right) \subseteq$ $\operatorname{im}\left(f_{m}\right)$ and $\operatorname{ker}\left(f_{n}\right) \subseteq \operatorname{ker}\left(\alpha_{n}^{m}\right)$. A sequence of strict homomorphisms

$$
\left\{M_{n}, \alpha_{n}\right\} \stackrel{\left\{f_{n}\right\}}{\longrightarrow}\left\{M_{n}^{\prime}, \alpha_{n}^{\prime}\right\} \stackrel{g_{n}}{\longrightarrow}\left\{M_{n}^{\prime \prime}, \alpha_{n}^{\prime \prime}\right\}
$$

will be called exact if the sequences of $R$-modules $M_{n} \stackrel{f_{n}}{\longrightarrow} N_{n} \stackrel{g_{n}}{\longrightarrow} M_{n}^{\prime \prime}$ is exact for each $n \geq 1$, and it is called pro-exact if $g_{n} \circ f_{n}=0$ holds for $n \geq 1$ and the pro- $R$-module $\left\{\operatorname{ker}\left(g_{n}\right) / \operatorname{im}\left(f_{n}\right)\right\}$ is pro-trivial.

The following results will be needed later.

Lemma 2.1. Let $0 \rightarrow\left\{M_{n}^{\prime}, \alpha_{n}^{\prime}\right\} \stackrel{\left\{f_{n}\right\}}{\longrightarrow}\left\{M_{n}, \alpha_{n}\right\} \stackrel{\left\{g_{n}\right\}}{\longrightarrow}\left\{M_{n}^{\prime \prime}, \alpha_{n}^{\prime \prime}\right\} \rightarrow 0$ be a pro-exact sequence of pro-R-modules. Then there is a natural exact sequence

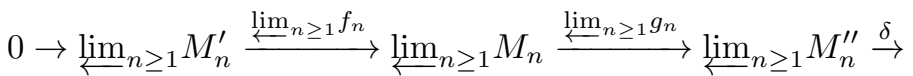

$$
\begin{aligned}
& \varliminf_{n \geq 1}^{1} M_{n}^{\prime} \stackrel{\lim _{n \geq 1}^{1} f_{n}}{\longleftrightarrow} \varliminf_{n \geq 1}^{1} M_{n} \stackrel{\lim _{n \geq 1}^{1} g_{n}}{\longleftrightarrow} \lim _{n \geq 1}^{1} M_{n}^{\prime \prime} \rightarrow 0 .
\end{aligned}
$$

In particular a pro-isomorphism $\left\{f_{n}\right\}:\left\{M_{n}, \alpha_{n}\right\} \rightarrow\left\{N_{n}, \beta_{n}\right\}$ induces isomorphisms

$$
\begin{array}{lll}
\varliminf_{n \geq 1} f_{n}: & \lim _{n \geq 1} M_{n} \stackrel{\cong}{\leftrightarrows} \lim _{n \geq 1} N_{n} ; \\
\varliminf_{n \geq 1}^{1} f_{n}: & \lim _{n \geq 1}^{1} M_{n} \stackrel{\cong}{\leftrightarrows} \lim _{n \geq 1}^{1} N_{n} .
\end{array}
$$

Proof. If $0 \rightarrow\left\{M_{n}^{\prime}, \alpha_{n}^{\prime}\right\} \stackrel{\left\{f_{n}\right\}}{\longrightarrow}\left\{M_{n}, \alpha_{n}\right\} \stackrel{g_{n}}{\longrightarrow}\left\{M_{n}^{\prime \prime}, \alpha_{n}^{\prime \prime}\right\} \rightarrow 0$ is exact, the construction of the six-term sequence is standard (see for instance [29, Proposition 7.63 on page 127]). Hence it remains to show for a pro-trivial pro- $R$-module $\left\{M_{n}, \alpha_{n}\right\}$ that $\varliminf_{n \geq 1} M_{n}$ and $\lim _{n \geq 1}^{1} M_{n}$ vanish. This follows directly from the standard construction for these limits as the kernel and cokernel of the homomorphism

$$
\prod_{n \geq 1} M_{n} \rightarrow \prod_{n \geq 1} M_{n}, \quad\left(x_{n}\right)_{n \geq 1} \mapsto\left(x_{n}-\alpha_{n+1}\left(x_{n+1}\right)\right)_{n \geq 1} .
$$


Lemma 2.2. Fix any commutative Noetherian ring $R$, and any ideal $I \subseteq R$. Then for any exact sequence $M^{\prime} \rightarrow M \rightarrow M^{\prime \prime}$ of finitely generated $R$-modules, the sequence

$$
\left\{M^{\prime} / I^{n} M^{\prime}\right\} \rightarrow\left\{M / I^{n} M\right\} \rightarrow\left\{M^{\prime \prime} / I^{n} M^{\prime \prime}\right\}
$$

of pro-R-modules is pro-exact.

Proof. It suffices to prove this for a short exact sequence $0 \rightarrow M^{\prime} \rightarrow M \rightarrow$ $M^{\prime \prime} \rightarrow 0$. Regard $M^{\prime}$ as a submodule of $M$, and consider the exact sequence

$$
0 \rightarrow\left\{\frac{\left(I^{n} M\right) \cap M^{\prime}}{I^{n} M^{\prime}}\right\} \rightarrow\left\{M^{\prime} / I^{n} M^{\prime}\right\} \rightarrow\left\{M / I^{n} M\right\} \rightarrow\left\{M^{\prime \prime} / I^{n} M^{\prime \prime}\right\} \rightarrow 0 .
$$

By [5, Theorem 10.11 on page 107], the filtrations $\left\{\left(I^{n} M\right) \cap M^{\prime}\right\}$ and $\left\{I^{n} M^{\prime}\right\}$ of $M^{\prime}$ have "bounded difference", i.e. there exists $k>0$ with the property that $\left(I^{n+k} M\right) \cap M^{\prime} \subseteq I^{n} M^{\prime}$ holds for all $n \geq 1$. The first term in the above exact sequence is thus pro-trivial, and so the remaining terms define a short sequence of pro- $R$-modules which is pro-exact.

\section{The K-Theory of the Classifying Space of a Finite Group}

Next we investigate the contravariant functor from the category FGINJ of finite groups with injective group homomorphisms as morphisms to the category $\mathbb{Z}$ - MOD of $\mathbb{Z}$-modules

$$
\mathrm{FGINJ} \rightarrow \mathbb{Z}-\mathrm{MOD}, \quad H \mapsto K^{k}(B H) .
$$

We need some input from representation theory. Recall that $R(G)$ denotes the complex representation ring. Let $\mathbb{I}_{G}$ be the kernel of the ring homomorphism $\operatorname{res}_{G}^{\{1\}}: R(G) \rightarrow R(\{1\}$ which is the same as the kernel of augmentation ring homomorphism $R(G) \rightarrow \mathbb{Z}$ sending $[V]$ to $\operatorname{dim}_{\mathbb{C}}(V)$. We will frequently use the so called double coset formula (see [26, Proposition 22 in Chapter 7 on page 58]). It says for two subgroups $H, K \subseteq G$

$$
\operatorname{res}_{G}^{K} \circ \operatorname{ind}_{H}^{G}=\sum_{K g H \in K \backslash G / H} \operatorname{ind}_{c(g): H \cap g^{-1} K g \rightarrow K} \circ \operatorname{res}_{H}^{H \cap g^{-1} K g},
$$

where $c(g)$ is conjugation with $g$, i.e. $c(g)(h)=g h g^{-1}$, and ind and res denote induction and restriction. One consequence of it is that $\operatorname{ind}_{H}^{G}: R(H) \rightarrow R(G)$ sends $\mathbb{I}_{H}$ to $\mathbb{I}_{G}$. Obviously $\operatorname{res}_{G}^{H}: R(G) \rightarrow R(H)$ maps $\mathbb{I}_{G}$ to $\mathbb{I}_{H}$.

For an abelian group $M$ let $M_{(p)}$ be the localization of $M$ at $p$. If $\mathbb{Z}_{(p)}$ is the subring of $\mathbb{Q}$ obtained from $\mathbb{Z}$ by inverting all prime numbers except $p$, then $M_{(p)}=M \otimes_{\mathbb{Z}} \mathbb{Z}_{(p)}$. Recall that the functor ? $\otimes_{\mathbb{Z}} \mathbb{Z}_{(p)}$ is exact. 
Lemma 3.2. Let $G$ be a finite group. Let $p$ be a prime number and denote by $G_{p}$ a p-Sylow subgroup of $G$. Then the composite

$$
R\left(G_{p}\right)_{(p)} \stackrel{\operatorname{ind}_{G}^{G}}{\longrightarrow} R(G)_{(p)} \stackrel{\operatorname{res}_{G}^{G}}{\longrightarrow} R\left(G_{p}\right)_{(p)}
$$

has the same image as

$$
\operatorname{res}_{G}^{G_{p}}: R(G)_{(p)} \rightarrow R\left(G_{p}\right)_{(p)} .
$$

Proof. A subgroup $H \subseteq G$ is called p-elementary if it is isomorphic to $C \times P$ for a cyclic group $C$ of order prime to $p$ and a $p$-group $P$. Let $\left\{C_{i} \times P_{i} \mid i=1,2, \ldots, r\right\}$ be a complete system of representatives of conjugacy classes of $p$-elementary subgroups of $G$. We can assume without loss of generality $P_{i} \subseteq G_{p}$. Define for $i=1,2, \ldots, r$ a homomorphism of abelian groups

$$
\phi_{i}:=\sum_{\substack{G_{p} \cdot g \cdot\left(C_{i} \times P_{i}\right) \in \\ G_{p} \backslash G /\left(C_{i} \times P_{i}\right)}} \operatorname{ind}_{c(g): P_{i} \cap g^{-1} G_{p} g \rightarrow G_{p}} \circ \operatorname{res}_{P_{i}}^{P_{i} \cap g^{-1} G_{p} g}: R\left(P_{i}\right) \rightarrow R\left(G_{p}\right) .
$$

Since the order of $C_{i}$ is prime to $p$, we have $\left(C_{i} \times P_{i}\right) \cap g^{-1} G_{p} g=P_{i} \cap g^{-1} G_{p} g$ for $g \in G$. Hence the following diagram commutes (actually before localization) by the double coset formula

$$
\begin{array}{rrr}
\bigoplus_{i=1}^{r} R\left(P_{i}\right)_{(p)} & \stackrel{\oplus_{i=1}^{r} \operatorname{ind}_{P_{i}}^{G_{p}}}{\longrightarrow} & R\left(G_{p}\right)_{(p)} \\
\oplus_{i=1}^{r} \operatorname{ind}_{P_{i}}^{G_{i} \times P_{i}} \downarrow & \downarrow^{\operatorname{ind}_{G_{p}}^{G}} \\
\bigoplus_{i=1}^{r} R\left(C_{i} \times P_{i}\right)_{(p)} \stackrel{\bigoplus_{i=1}^{r} \operatorname{ind}_{C_{i} \times P_{i}}^{G_{p}}}{\longrightarrow} & R(G)_{(p)} \\
\oplus_{i=1}^{r} \operatorname{res}_{C_{i} \times P_{i}}^{P_{i}} \downarrow & \downarrow \operatorname{res}_{G}^{G_{p}} \\
\bigoplus_{i=1}^{r} R\left(P_{i}\right)_{(p)} & \stackrel{\oplus_{i=1}^{r} \phi_{i}}{\longrightarrow} & R\left(G_{p}\right)_{(p)}
\end{array}
$$

The middle horizontal arrow $\bigoplus_{i=1}^{r} \operatorname{ind}_{C_{i} \times P_{i}}^{G_{p}}$ is surjective by Brauer's Theorem [26, Theorem 18 in Chapter 10 on page 75]. The composite of the left lower vertical arrow and the left upper vertical arrow $\bigoplus_{i=1}^{r} \operatorname{res}_{C_{i} \times P_{i}}^{P_{i}} \circ \operatorname{ind}_{P_{i}}^{C_{i} \times P_{i}}$ is $\bigoplus_{i=1}^{r}\left|C_{i}\right| \cdot$ id and hence an isomorphism. Now the claim follows from an easy diagram chase.

Lemma 3.3. Let $p$ and $q$ be different primes. Then the composition

$$
R\left(G_{p}\right) \stackrel{\operatorname{ind}_{G_{p}}^{G}}{\longrightarrow} R(G) \stackrel{\operatorname{res}_{G}^{G_{q}}}{\longrightarrow} R\left(G_{q}\right)
$$

agrees with $\left|G_{q} \backslash G / G_{p}\right| \cdot \operatorname{ind}_{\{1\}}^{G_{q}} \circ \operatorname{res}_{G_{p}}^{\{1\}}$.

Proof. This follows from the double coset formula (3.1) since $G_{p} \cap g^{-1} G_{q} g=\{1\}$ for each $g \in G$. 
Lemma 3.4. Let $G$ be a finite group and let $\mathbb{I}_{G} \subseteq R(G)$ be the augmentation ideal. Then the following sequence of $R(G)$-modules is exact

$$
0 \rightarrow \bigcap_{m \geq 1}\left(\mathbb{I}_{G}\right)^{m} \stackrel{i}{\rightarrow} \mathbb{I}_{G} \stackrel{\prod_{p} \operatorname{res}_{G}^{G_{p}}}{\rightarrow} \prod_{p \in \mathcal{P}(G)} \operatorname{im}\left(\operatorname{res}_{G}^{G_{p}}: \mathbb{I}_{G} \rightarrow \mathbb{I}_{G_{p}}\right) \rightarrow 0
$$

where $i$ is the inclusion and $\mathcal{P}(G)$ is the set of primes dividing $|G|$.

Proof. The kernel of $\prod_{p} \operatorname{res}_{G}^{G_{p}}: R(G) \rightarrow \prod_{p} R\left(G_{p}\right)$ is $\bigcap_{m \geq 1}\left(\mathbb{I}_{G}\right)^{m}$ by [4, Proposition 6.12 on page 269]. Hence it remains to show that

$$
\prod_{p} \operatorname{res}_{G}^{G_{p}}: \mathbb{I}_{G} \rightarrow \prod_{p} \operatorname{im}\left(\operatorname{res}_{G}^{G_{p}}: \mathbb{I}_{G} \rightarrow \mathbb{I}_{G_{p}}\right)
$$

is surjective. It suffices to show for a each prime number $q$ that its localization

$$
\prod_{p} \operatorname{res}_{G}^{G_{p}}:\left(\mathbb{I}_{G}\right)_{(q)} \rightarrow \prod_{p} \operatorname{im}\left(\operatorname{res}_{G}^{G_{p}}:\left(\mathbb{I}_{G}\right)_{(q)} \rightarrow\left(\mathbb{I}_{G_{p}}\right)_{(q)}\right)
$$

is surjective. Next we construct the following commutative diagram

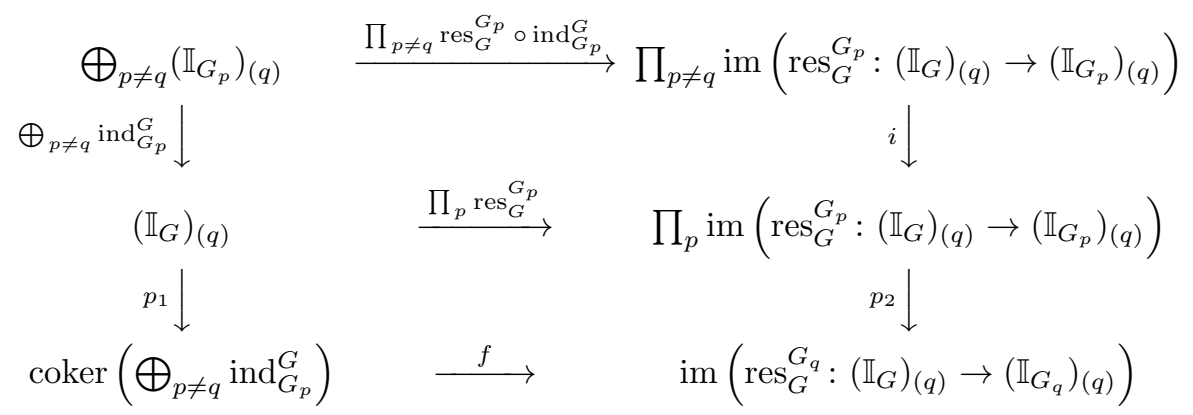

Here $i$ is the inclusion and $p_{1}$ and $p_{2}$ are the obvious projections. Since the composition

$$
\begin{aligned}
\bigoplus_{p \neq q}\left(\mathbb{I}_{G_{p}}\right)_{(q)} \stackrel{\oplus_{p \neq q} \operatorname{ind}_{G_{p}}^{G}}{\longrightarrow}\left(\mathbb{I}_{G}\right)_{(q)} \stackrel{\prod_{p} \operatorname{res}_{G}^{G_{p}}}{\longrightarrow} \prod_{p} \operatorname{im}\left(\operatorname{res}_{G}^{G_{p}}:\left(\mathbb{I}_{G}\right)_{(q)} \rightarrow\left(\mathbb{I}_{G_{p}}\right)_{(q)}\right) \\
\stackrel{p_{2}}{\longrightarrow} \operatorname{im}\left(\operatorname{res}_{G}^{G_{q}}:\left(\mathbb{I}_{G}\right)_{(q)} \rightarrow\left(\mathbb{I}_{G_{q}}\right)_{(q)}\right)
\end{aligned}
$$

agrees with

$$
\bigoplus_{p \neq q} \operatorname{res}_{G}^{G_{q}} \circ \operatorname{ind}_{G_{p}}^{G}: \bigoplus_{p \neq q}\left(\mathbb{I}_{G_{p}}\right)_{(q)} \rightarrow \operatorname{im}\left(\operatorname{res}_{G}^{G_{q}}:\left(\mathbb{I}_{G}\right)_{(q)} \rightarrow\left(\mathbb{I}_{G_{q}}\right)_{(q)}\right)
$$

and hence is trivial by Lemma 3.3, there exists a map

$$
f: \operatorname{coker}\left(\bigoplus_{p \neq q} \operatorname{ind}_{G_{p}}^{G}\right) \rightarrow \operatorname{im}\left(\operatorname{res}_{G}^{G_{q}}:\left(\mathbb{I}_{G}\right)_{(q)} \rightarrow\left(\mathbb{I}_{G_{q}}\right)_{(q)}\right)
$$


such that the diagram above commutes. Since

$$
p_{2} \circ \prod_{p} \operatorname{res}_{G}^{G_{p}}=\operatorname{res}_{G}^{G_{q}}:\left(\mathbb{I}_{G}\right)_{(q)} \rightarrow \operatorname{im}\left(\operatorname{res}_{G}^{G_{q}}:\left(\mathbb{I}_{G}\right)_{(q)} \rightarrow\left(\mathbb{I}_{G_{q}}\right)_{(q)}\right)
$$

is by definition surjective, $f$ is surjective. The upper horizontal arrow in the commutative diagram above is surjective by Lemma 3.2. Now the claim follows by an easy diagram chase.

Theorem 3.5 (Structure of $\left.\left\{\mathbb{I}_{G} /\left(\mathbb{I}_{G}\right)^{n+1}\right\}\right)$. Let $G$ be a finite group. Let $\mathcal{P}(G)$ be the set of primes dividing $|G|$.

(a) There are positive integers $a, b$ and $c$ such that for each prime $p$ dividing the order of $|G|$

$$
\begin{aligned}
p^{a} \cdot \mathbb{I}_{G_{p}} & \subseteq \mathbb{I}_{G_{p}}^{2} ; \\
\mathbb{I}_{G_{p}}^{b} & \subseteq p \cdot \mathbb{I}_{G_{p}} ; \\
\mathbb{I}_{G} \cdot \mathbb{I}_{G_{p}} & \subseteq \mathbb{I}_{G_{p}}^{2} ; \\
\left(\mathbb{I}_{G_{p}}\right)^{c} & \subseteq \mathbb{I}_{G} \cdot \mathbb{I}_{G_{p}} ;
\end{aligned}
$$

(b) For a prime $p$ dividing $|G|$ let $\operatorname{im}\left(\operatorname{res}_{G}^{G_{p}}\right)$ be the image of $\operatorname{res}_{G}^{G_{p}}: \mathbb{I}_{G} \rightarrow \mathbb{I}_{G_{p}}$. We obtain a sequence of pro-isomorphisms of pro-Z-Z-modules

$$
\begin{aligned}
\left\{\mathbb{I}_{G} /\left(\mathbb{I}_{G}\right)^{n+1}\right\} & \cong \prod_{p \in \mathcal{P}(G)}\left\{\operatorname{im}\left(\operatorname{res}_{G}^{G_{p}}\right) /\left(\mathbb{I}_{G}\right)^{n} \cdot \operatorname{im}\left(\operatorname{res}_{G}^{G_{p}}\right)\right\} \\
& \cong \prod_{p \in \mathcal{P}(G)}\left\{\operatorname{im}\left(\operatorname{res}_{G}^{G_{p}}\right) /\left(\mathbb{I}_{G_{p}}\right)^{n} \cdot \operatorname{im}\left(\operatorname{res}_{G}^{G_{p}}\right)\right\} \\
& \cong \prod_{p \in \mathcal{P}(G)}\left\{\operatorname{im}\left(\operatorname{res}_{G}^{G_{p}}\right) /\left(\mathbb{I}_{G_{p}}\right)^{b n} \cdot \operatorname{im}\left(\operatorname{res}_{G}^{G_{p}}\right)\right\} \\
& \cong \prod_{p \in \mathcal{P}(G)}\left\{\operatorname{im}\left(\operatorname{res}_{G}^{G_{p}}\right) / p^{n} \cdot \operatorname{im}\left(\operatorname{res}_{G}^{G_{p}}\right)\right\} .
\end{aligned}
$$

(c) There is an isomorphism of pro-Z-Z-modules

$$
\{\mathbb{Z}\} \oplus\left\{\mathbb{I}_{G} /\left(\mathbb{I}_{G}\right)^{n}\right\} \stackrel{\cong}{\longrightarrow}\left\{R(G) /\left(\mathbb{I}_{G}\right)^{n}\right\},
$$

where $\{\mathbb{Z}\}$ denotes the constant inverse system $\mathbb{Z} \stackrel{\text { id }}{\longleftarrow} \mathbb{Z} \stackrel{\text { id }}{\longleftarrow} \ldots$

Proof. (a) The existence of the integers $a, b$ and $c$ for which the inclusions appearing in the statement of Theorem 3.5 hold follows from results of [4, Theorem 6.1 on page 265] and [7, Proposition 1.1 in Part III on page 277].

(b) These inequalities of assertion (a) imply that the second, third and fourth 
map of pro- $\mathbb{Z}$-isomorphism appearing in the statement of Theorem 3.5 are indeed well-defined pro-isomorphisms. The first map

$$
\left\{\mathbb{I}_{G} /\left(\mathbb{I}_{G}\right)^{n+1}\right\} \stackrel{\cong}{\longrightarrow} \prod_{p \in \mathcal{P}(G)}\left\{\operatorname{im}\left(\operatorname{res}_{G}^{G_{p}}\right) /\left(\mathbb{I}_{G}\right)^{n} \cdot \operatorname{im}\left(\operatorname{res}_{G}^{G_{p}}\right)\right\}
$$

is a well-defined pro-isomorphism of pro- $\mathbb{Z}$-modules by Lemma 2.2 and Lemma 3.4 provided $\left\{\left(\bigcap_{m \geq 1}\left(\mathbb{I}_{G}\right)^{m}\right) / \mathbb{I}_{G}^{n} \cdot\left(\bigcap_{m \geq 1}\left(\mathbb{I}_{G}\right)^{m}\right)\right\}$ is pro-trivial. The latter statement follows from Lemma 2.2 applied to the exact sequence

$$
0 \quad \rightarrow \quad \bigcap_{m \geq 1}\left(\mathbb{I}_{G}\right)^{m} \quad \rightarrow \quad \mathbb{I}_{G} \quad \rightarrow \quad \mathbb{I}_{G} / \bigcap_{m \geq 1}\left(\mathbb{I}_{G}\right)^{m} \rightarrow \quad \rightarrow \quad 0
$$

(c) Consider the isomorphism of finitely generated free abelian groups

$$
\mathbb{Z} \oplus \mathbb{I}_{G} \cong R(G), \quad(m, x) \mapsto x+m \cdot[\mathbb{C}]
$$

It becomes an isomorphism of rings if we equip the source with the multiplication $(m, x) \cdot(n, y)=(m n, m y+n x+x y)$. In particular $\mathbb{I}_{G}^{n} \cdot\left(\mathbb{I}_{G} \oplus \mathbb{Z}\right) \subseteq \mathbb{I}_{G}^{n} \oplus 0$ for $n \geq 1$. This finishes the proof of Theorem 3.5.

Now we can give the proof of Theorem 0.3 .

Proof. In the sequel we abbreviate $\operatorname{im}\left(\operatorname{res}_{G}^{G_{p}}\right)=\operatorname{im}\left(\operatorname{res}_{G}^{G_{p}}: \mathbb{I}_{G} \rightarrow \mathbb{I}_{G_{p}}\right)$. Notice that $\operatorname{im}\left(\operatorname{res}_{G}^{G_{p}}\right) \subseteq R\left(G_{p}\right)$ is a finitely generated free $\mathbb{Z}$-module. We obtain from Lemma 2.1 and Theorem 3.5 an isomorphism

$$
\lim _{n \geq 1} R(G) /\left(\mathbb{I}_{G}\right)^{n} \cong \mathbb{Z} \times \prod_{p \in \mathcal{P}(G)} \lim _{n \geq 1} \operatorname{im}\left(\operatorname{res}_{G}^{G_{p}}\right) / p^{n} \cdot \operatorname{im}\left(\operatorname{res}_{G}^{G_{p}}\right) .
$$

Now the Atiyah-Segal Completion Theorem [6] yields an isomorphisms

$$
\lim _{n \geq 1} R(G) /\left(\mathbb{I}_{G}\right)^{n} \cong \lim _{n \geq 1} K^{0}\left((B G)_{n}\right) \stackrel{\cong}{\longleftarrow} K^{0}(B G)
$$

and $K^{1}(B G)=0$. This implies

$$
\begin{aligned}
K^{0}(B G) \cong \mathbb{Z} \oplus \bigoplus_{p \in \mathcal{P}(G)} \operatorname{im}\left(\operatorname{res}_{G}^{G_{p}}: \mathbb{I}_{G} \rightarrow \mathbb{I}_{G_{p}}\right) \otimes_{\mathbb{Z}} \mathbb{Z}_{p} \\
K^{1}(B G) \cong 0 .
\end{aligned}
$$

Next we show that the rank of the finitely generated free abelian group $\operatorname{im}\left(\operatorname{res}_{G}^{G_{p}}: \mathbb{I}_{G} \rightarrow \mathbb{I}_{G_{p}}\right) \subseteq R\left(G_{p}\right)$ is the number $r(p)$ of conjugacy classes $(g)$ of elements $g \in G$ whose order $|g|$ is $p^{d}$ for some integer $d \geq 1$. This follows from the commutative diagram 


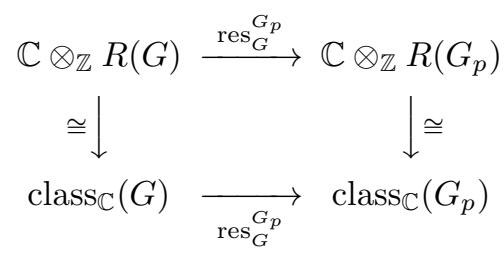

where $\operatorname{class}_{\mathbb{C}}(G)$ denotes the complex vector space of class functions on $G$, i.e. functions $G \rightarrow \mathbb{C}$ which are constant on conjugacy classes of elements, (and analogous for $G_{p}$ ), the vertical isomorphisms are given by taking the character of a complex representation, and the lower horizontal arrow is given by restricting a function $G \rightarrow \mathbb{C}$ to $G_{p}$.

Recall that $\mathbb{I}_{p}(G)$ is canonically isomorphic to $\operatorname{im}\left(\operatorname{res}_{G}^{G_{p}}: \mathbb{I}_{G} \rightarrow \mathbb{I}_{G_{p}}\right)$.

One easily checks that the isomorphisms obtained from the one appearing in Theorem 3.5 (b) and (c) by applying the inverse limit and the isomorphism (3.6) are compatible with the obvious multiplicative structures.

This finishes the proof of Theorem 0.3.

\section{Proof of the Main Result}

In this section we want to prove our main Theorem 0.1. We want to apply the cohomological equivariant Chern character of [20] to the equivariant cohomology theory $H_{?}^{*}\left(-;\left(\mathbf{K}_{\mathrm{Bor}}\right)_{(0)}\right)$. This requires to analyze the contravariant functor

$$
\text { FGINJ } \rightarrow \mathbb{Q} \text {-MOD, } \quad H \mapsto H_{G}^{l}\left(G / H ;\left(\mathbf{K}_{\mathrm{Bor}}\right)_{(0)}\right) .
$$

From (1.2) and Lemma 1.6 we conclude that the contravariant functor (4.1) is naturally equivalent to the contravariant functor

$$
\text { FGINJ } \rightarrow \mathbb{Q}-\text { MOD }, \quad H \mapsto K^{l}(B H) \otimes_{\mathbb{Z}} \mathbb{Q} .
$$

Theorem 0.3 yields the contravariant functor (4.2) is trivial for odd $l$ and is naturally equivalent to the contravariant functor

$$
\text { FGINJ } \rightarrow \mathbb{Q}-\mathrm{MOD} \quad H \mapsto \mathbb{Q} \times \prod_{p} \mathbb{I}_{p}(H) \otimes_{\mathbb{Z}} \mathbb{Q}_{p}^{-}
$$

for even $l$, where the factor $\mathbb{Q}$ is constant in $H$ and functoriality for the other factors is given by restriction.

Given a contravariant functor $F:$ FGINJ $\rightarrow \mathbb{Q}$ - MOD, define the $\mathbb{Q}[\operatorname{aut}(H)]$ module

$$
T_{H} F(H):=\operatorname{ker}\left(\prod_{K \subsetneq H} F(K \hookrightarrow H): F(H) \rightarrow \prod_{K \subsetneq H} F(K)\right) .
$$


Next we compute $T_{H}\left(K^{0}(B H) \otimes_{\mathbb{Z}} \mathbb{Q}\right)$. Since $T_{H}$ is compatible with direct products, we obtain from (4.3) a canonical $\mathbb{Q}[\operatorname{aut}(H)]$-isomorphism

$$
T_{H}\left(K^{0}(B H) \otimes_{\mathbb{Z}} \mathbb{Q}\right)=T_{H}(\mathbb{Q}) \times \prod_{p} T_{H}\left(\mathbb{I}_{p}(H) \otimes_{\mathbb{Z}} \mathbb{Q}_{p}\right) .
$$

Since $\mathbb{Q}$ is the constant functor, we get

$$
T_{H}(\mathbb{Q}):= \begin{cases}0 & \text { if } H \neq\{1\} \\ \mathbb{Q} & \text { if } H=\{1\} .\end{cases}
$$

Fix a prime number $p$. Since for any finite group $H$ the map given by restriction to finite cyclic subgroups

$$
R(H) \rightarrow \prod_{\substack{C \subseteq H \\ C \text { cyclic }}} R(C)
$$

is injective, we conclude

Lemma 4.7. For a finite group $H$

$$
T_{H}\left(\mathbb{I}_{p}(H)\right)=0,
$$

unless $H$ is a non-trivial cyclic p-group.

Let $C$ be a non-trivial finite cyclic $p$-group. Then we get

$$
T_{C}\left(\mathbb{I}_{p}(C)\right)=\operatorname{ker}\left(\operatorname{res}_{C}^{C^{\prime}}: R(C) \rightarrow R\left(C^{\prime}\right)\right),
$$

where $C^{\prime} \subseteq C$ is the unique cyclic subgroup of index $p$ in $C$.

Recall that taking the character of a rational representation of a finite group $H$ yields an isomorphism

$$
\chi: R_{\mathbb{Q}}(H) \otimes_{\mathbb{Z}} \mathbb{Q} \stackrel{\cong}{\rightarrow} \operatorname{class}_{\mathbb{Q}}(H),
$$

where $R_{\mathbb{Q}}(C)$ is the rational representation ring of $C$ and $\operatorname{class}_{\mathbb{Q}}(H)$ is the rational vector space of functions $f: H \rightarrow \mathbb{Q}$ for which $f\left(g_{1}\right)=f\left(g_{2}\right)$ holds if the cyclic subgroups generated by $g_{1}$ and $g_{2}$ are conjugate in $H$ (see [26, page 68 and Theorem 29 on page 102]). Hence there is an idempotent $\theta_{C} \in R_{\mathbb{Q}}(C) \otimes_{\mathbb{Z}} \mathbb{Q}$ which is uniquely determined by the property that its character sends a generator of $C$ to 1 and all other elements to 0 . Denote its image under the change of coefficients map $R_{\mathbb{Q}}(C) \otimes_{\mathbb{Z}} \mathbb{Q} \rightarrow R(C) \otimes_{\mathbb{Z}} \mathbb{Q}$ also by $\theta_{C}$. Let $\theta_{C} \cdot R(C) \otimes_{\mathbb{Z}} \mathbb{Q}_{p}^{-} \subseteq R(C) \otimes_{\mathbb{Z}} \mathbb{Q}_{p}$ be the image of the idempotent endomorphism $R(C) \otimes_{\mathbb{Z}} \mathbb{Q}_{p} \rightarrow R(C) \otimes_{\mathbb{Z}} \mathbb{Q}_{p}$ given by multiplication with $\theta_{C}$.

Lemma 4.9. For every non-trivial cyclic p-group $C$ the inclusion induces a $\mathbb{Q}[\operatorname{aut}(C)]$-isomorphism

$$
\theta_{C} \cdot R(C) \otimes_{\mathbb{Z}} \mathbb{Q} \stackrel{\cong}{\rightrightarrows} T_{C}\left(\mathbb{I}_{p}(C) \otimes_{\mathbb{Z}} \mathbb{Q}\right) .
$$


Proof. Since the map $\operatorname{res}_{C}^{C^{\prime}}: R(C) \otimes_{\mathbb{Z}} \mathbb{Q} \rightarrow R\left(C^{\prime}\right) \otimes_{\mathbb{Z}} \mathbb{Q}$ sends $\theta_{C}$ to zero, $\theta_{C} \cdot R(C) \otimes_{\mathbb{Z}} \mathbb{Q}$ is contained in $\operatorname{ker}\left(\operatorname{res}_{C}^{C^{\prime}}: R(C) \rightarrow R\left(C^{\prime}\right)\right) \otimes_{\mathbb{Z}} \mathbb{Q}$. For $x \in$ $\operatorname{ker}\left(\operatorname{res}_{C}^{C^{\prime}}: R(C) \rightarrow R\left(C^{\prime}\right)\right) \otimes_{\mathbb{Z}} \mathbb{Q}$ one gets $\theta_{C} \cdot x-x=0$ by the calculation appearing in the proof of [19, Lemma $3.4(\mathrm{~b})]$.

Lemma 4.10. For every proper $G-C W$-complex $X$ and $n \in \mathbb{Z}$ there is an isomorphism, natural in $X$,

$$
\begin{aligned}
& \overline{\mathrm{ch}}_{G}^{n}: H_{G}^{*}\left(X ;\left(\mathbf{K}_{\text {Bor }}\right)_{(0)}\right) \stackrel{\cong}{\longrightarrow} \\
& \prod_{i \in \mathbb{Z}} H^{2 i+n}(G \backslash X ; \mathbb{Q}) \times \prod_{p} \prod_{(C) \in \mathcal{C}_{p}(G)} \prod_{i \in \mathbb{Z}} H_{W_{G} C}^{2 i+n}\left(C_{G} C \backslash X^{C} ; \theta_{C} \cdot R(C) \otimes_{\mathbb{Z}} \mathbb{Q}_{p}\right),
\end{aligned}
$$

where $\mathcal{C}_{p}(C)$ is the set of conjugacy classes of non-trivial cyclic p-subgroups of $G$ and $W_{G} C=N_{G} C / C_{G} C$ is considered as a subgroup of aut $(C)$ and thus acts on $\theta_{C} \cdot R(C) \otimes_{\mathbb{Z}} \mathbb{Q}_{p}$.

Proof. This follows from [20, Theorem 5.5 (c) and Example 5.6] using (4.6), Lemma 4.7 and Lemma 4.9 .

For a generator $t \in C$ let $\mathbb{C}_{t}$ be the $\mathbb{C}$-representation with $\mathbb{C}$ as underlying complex vector space such that $t$ operates on $\mathbb{C}$ by multiplication with $\exp \left(\frac{2 \pi i}{|C|}\right)$. Let $\operatorname{Gen}(C)$ be the set of generators. Notice that aut $(C)$ acts in an obvious way on $\operatorname{Gen}(C)$ such that the aut $(C)$-action is transitive and free, and acts on $R(C)$ by restriction. In the sequel $\chi_{V}$ denotes for a complex representation $V$ its character.

Lemma 4.11. Let $C$ be a finite cyclic group. Then

(a) The map

$$
v(C): \theta_{C} \cdot R(C) \otimes_{\mathbb{Z}} \mathbb{C} \stackrel{\cong}{\longrightarrow} \prod_{\operatorname{Gen}(C)} \mathbb{C}, \quad[V] \mapsto\left(\chi_{V}(t)\right)_{t \in \operatorname{Gen}(C)}
$$

is a $\mathbb{C}[\operatorname{aut}(C)]$-isomorphism if aut $(C)$ acts on the target by permuting the factors. The map $v(C)$ is compatible with the ring structure on the source induced by the tensor product of representations and the product ring structure on the target;

(b) There is an isomorphism of $\mathbb{Q}[\operatorname{aut}(C)]$-modules

$$
u(C): \mathbb{Q}[\operatorname{Gen}(C)] \stackrel{\cong}{\Rightarrow} \theta_{C} \cdot R(C) \otimes_{\mathbb{Z}} \mathbb{Q} .
$$

Proof. (a) The map

$$
R(C) \otimes_{\mathbb{Z}} \mathbb{C} \stackrel{\cong}{\rightarrow} \prod_{g \in C} \mathbb{C}, \quad[V] \mapsto\left(\chi_{V}(g)\right)_{g \in C}
$$


is an isomorphism of rings. One easily checks that it is compatible with the $\operatorname{aut}(C)$ actions. Now the assertion follows from the fact that the character of $\theta_{C}$ sends a generator of $C$ to 1 and any other element of $C$ to 0 .

(b) Obviously $\mathbb{Q}[\operatorname{Gen}(C)]$ is $\mathbb{Q}[\operatorname{aut}(C)]$-isomorphic to the regular representation $\mathbb{Q}[\operatorname{aut}(C)]$ since $\operatorname{Gen}(C)$ is a transitive free aut $(C)$-set. It remains to show that $\theta_{C} \cdot R(C) \otimes_{\mathbb{Z}} \mathbb{Q}$ is $\mathbb{Q}[\operatorname{aut}(C)]$-isomorphic to the regular representation $\mathbb{Q}[\operatorname{aut}(C)]$. By character theory it suffices to show that $\theta_{C} \cdot R(C) \otimes_{\mathbb{Z}} \mathbb{C}$ is $\mathbb{C}[\operatorname{aut}(C)]$-isomorphic to the regular representation $\mathbb{C}[\operatorname{aut}(C)]$. This follows from assertion (a).

Lemma 4.12. For every proper $G-C W$-complex $X$ and $n \in \mathbb{Z}$ there is an isomorphism, natural in $X$,

$$
\begin{aligned}
\overline{\overline{\mathrm{ch}}}_{G}^{n}: H_{G}^{*}\left(X ;\left(\mathbf{K}_{\mathrm{Bor}}\right)_{(0)}\right) \stackrel{\cong}{\rightrightarrows} & \\
& \prod_{i \in \mathbb{Z}} H^{2 i+n}(G \backslash X ; \mathbb{Q}) \times \prod_{p} \prod_{(g) \in \operatorname{con}_{p}(G)} H^{2 i+n}\left(C_{G}\langle g\rangle \backslash X^{\langle g\rangle} ; \mathbb{Q}_{p}^{-}\right) .
\end{aligned}
$$

Proof. Fix a prime $p$. Let $C$ be a cyclic subgroup of $G$ of order $p^{d}$ for some integer $d \geq 1$. The obvious $N_{G} C$-action on $C$ given by conjugation induces an embedding of groups $W_{G} C \rightarrow \operatorname{aut}(C)$. The obvious action of aut $(C)$ on $\operatorname{Gen}(C)$ is free and transitive. Thus we obtain an isomorphism of $\mathbb{Q}_{p}\left[W_{G} C\right]$-modules

$$
\mathbb{Q}_{p}^{-}[\operatorname{Gen}(C)] \cong \prod_{W_{G} C \backslash \operatorname{Gen}(C)} \mathbb{Q}_{p}^{\wedge}\left[W_{G} C\right] .
$$

This induces a natural isomorphism

$$
H_{W_{G} C}^{k}\left(C_{G} C \backslash X^{C} ; \mathbb{Q}_{p}^{-}[\operatorname{Gen}(C)]\right) \stackrel{\cong}{\rightrightarrows} \prod_{W_{G} C \backslash \operatorname{Gen}(C)} H^{k}\left(C_{G} C \backslash X^{C} ; \mathbb{Q}_{p}^{-}\right),
$$

which comes from the adjunction $\left(i^{*}, i_{!}\right)$of the functor restriction $i^{*}$ and coin-

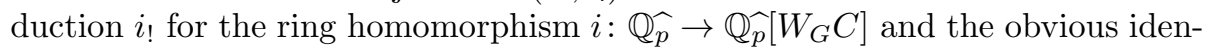
tification $i_{!}\left(\mathbb{Q}_{p}\right)=\mathbb{Q}_{p}\left[W_{G} C\right]$. There is an obvious bijection between the sets

$$
\coprod_{(C) \in \mathcal{C}_{p}} W_{G} C \backslash \operatorname{Gen}(C) \cong \operatorname{con}_{p}(G)
$$

Now the claim follows from Lemma 4.10 and Lemma 4.11 (b).

Theorem 4.13 (Computation of $K^{n}\left(E G \times_{G} X\right) \otimes_{\mathbb{Z}} \mathbb{Q}$ ). For every finite proper $G$-CW-complex $X$ and $n \in \mathbb{Z}$ there is a natural isomorphism

$$
\begin{aligned}
\overline{\operatorname{ch}}_{G}^{n}: K^{n} & \left(E G \times_{G} X\right) \otimes_{\mathbb{Z}} \mathbb{Q} \\
& \cong \prod_{i \in \mathbb{Z}} H^{2 i+n}(G \backslash X ; \mathbb{Q}) \times \prod_{p} \prod_{(g) \in \operatorname{con}_{p}(G)} H^{2 i+n}\left(C_{G}\langle g\rangle \backslash X^{\langle g\rangle} ; \mathbb{Q}_{p}^{-}\right) .
\end{aligned}
$$


Proof. This follows from Lemma 1.6 and Lemma 4.12 .

Lemma 4.14. Let $Y \neq \emptyset$ be a proper $G-C W$-complex such that $\widetilde{H}_{p}(Y ; \mathbb{Q})$ vanishes for all $p$. Let $f: Y \rightarrow \underline{E} G$ be a $G$-map. Then $G \backslash f: G \backslash Y \rightarrow G \backslash \underline{E} G$ induces for all $k$ isomorphisms

$$
\begin{aligned}
& H_{k}(G \backslash f ; \mathbb{Q}): H_{k}(G \backslash Y ; \mathbb{Q}) \stackrel{\cong}{\rightrightarrows} H_{k}(G \backslash \underline{E} G ; \mathbb{Q}) ; \\
& H^{k}(G \backslash f ; \mathbb{Q}): H^{k}(G \backslash \underline{E} G ; \mathbb{Q}) \stackrel{\cong}{\rightrightarrows} H^{k}(G \backslash Y ; \mathbb{Q}) ; \\
& H^{k}(G \backslash f ; \mathbb{C}): H^{k}(G \backslash \underline{E} G ; \mathbb{C}) \stackrel{\cong}{\rightrightarrows} H^{k}(G \backslash Y, \mathbb{C}) ; \\
& H^{k}\left(G \backslash f ; \mathbb{Q}_{p}\right): H^{k}\left(G \backslash \underline{E} G ; \mathbb{Q}_{p}^{-}\right) \stackrel{\cong}{\cong} H^{k}\left(G \backslash Y, \mathbb{Q}_{p}\right) ; \\
& H^{k}\left(G \backslash f ; \mathbb{Q}_{p}^{\hat{p}} \otimes_{\mathbb{Q}} \mathbb{C}\right): H^{k}\left(G \backslash \underline{E} G ; \mathbb{Q}_{p}^{\hat{p}} \otimes_{\mathbb{Q}} \mathbb{C}\right) \stackrel{\cong}{\rightrightarrows} H^{k}\left(G \backslash Y, \mathbb{Q}_{p}^{\wedge} \otimes_{\mathbb{Q}} \mathbb{C}\right) .
\end{aligned}
$$

Proof. The map $C_{*}(f) \otimes_{\mathbb{Z}} \operatorname{id}_{\mathbb{Q}}: C_{*}(G \backslash Y) \otimes_{\mathbb{Z}} \mathbb{Q} \rightarrow C_{*}(\underline{E} G) \otimes_{\mathbb{Z}} \mathbb{Q}$ is $\mathbb{Q}$-chain map of projective $\mathbb{Q} G$-chain complexes and induces an isomorphism on homology. Hence it is a $\mathbb{Q} G$-chain homotopy equivalence. This implies that $C_{*}(f) \otimes_{\mathbb{Q} G} M$ and $\operatorname{hom}_{\mathbb{Q} G}\left(C_{*}(f), M\right)$ are chain homotopy equivalences and induce isomorphisms on homology and cohomology respectively for every $\mathbb{Q}$-module $M$.

Now we can give the proof of Theorem 0.1.

Proof. We conclude from Lemma 4.14 that for any $g \in \operatorname{con}_{p}(G)$ the up to $C_{G}\langle g\rangle$-homotopy unique $C_{G}\langle g\rangle$-map $f_{g}: E C_{G}\langle g\rangle \rightarrow \underline{E} C_{G}\langle g\rangle$ and the up to $G$ homotopy unique $G$-map $f: E G \rightarrow \underline{E G}$ induce isomorphisms

$$
\begin{aligned}
H^{k}(G \backslash f ; \mathbb{Q}): H^{k}(G \backslash \underline{E} G ; \mathbb{Q}) & \cong H^{k}(B G ; \mathbb{Q}) ; \\
H^{k}\left(C_{G}\langle g\rangle \backslash f_{g} ; \mathbb{Q}_{p}\right): H^{k}\left(C_{G}\langle g\rangle \backslash \underline{E} C_{G}\langle g\rangle ; \mathbb{Q}_{p}\right) & \cong H^{k}\left(B C_{G}\langle g\rangle, \mathbb{Q}_{p}\right) .
\end{aligned}
$$

Now apply Theorem 4.13 to $X=\underline{E} G$ and use (4.15) and (4.16) together with the fact that $\underline{E} G^{\langle g\rangle}$ is a model for $\underline{E} C_{G}\langle g\rangle$.

\section{Multiplicative Structures}

In this section we want to deal with multiplicative structures and prove Theorem 0.2.

Remark 5.1. (Ring structures and multiplicative structures). Suppose that the $\Omega$-spectrum $\mathbf{E}$ comes with the structure of a ring spectrum $\mu: \mathbf{E} \wedge \mathbf{E} \rightarrow$ E. It induces a multiplicative structure on the (non-equivariant) cohomology theory $H^{*}(-; \mathbf{E})$ associated to $\mathbf{E}$. Thus the equivariant cohomology theory given by the equivariant Borel cohomology $H_{?}^{*}(E ? \times ?-; \mathbf{E})$ associated to $\mathbf{E}$ inherits a multiplicative structure the sense of $[20$, Section 6$]$.

If the contravariant GROUPOIDS- $\Omega$-spectrum $\mathbf{F}$ comes with a ring structure of contravariant GROUPOIDS- $\Omega$-spectra $\mu: \mathbf{F} \wedge \mathbf{F} \rightarrow \mathbf{F}$, then the associated 
equivariant cohomology theory $H_{?}^{*}(-; \mathbf{F})$ inherits a multiplicative structure. A ring structure on the $\Omega$-spectrum $\mathbf{E}$ induces a ring structure of contravariant GROUPOIDS- $\Omega$-spectra on $\mathbf{E}_{\text {Bor }}$. The induced multiplicative structure on $H_{?}^{*}\left(-; \mathbf{E}_{\text {Bor }}\right)$ and the one on $H_{?}^{*}(E ? \times$ ? $-; \mathbf{E})$ are compatible with the natural identification (1.2).

A ring structure on the $\Omega$-spectrum $\mathbf{E}$ induces in a natural way a ring structure on its rationalization $\operatorname{Rat}(\mathbf{E})$. Thus a ring structure on the contravariant GROUPOIDS- $\Omega$-spectra on $\mathbf{E}_{\text {Bor }}$ induces a ring structure on the contravariant GROUPOIDS- $\Omega$-spectra on $\left(\mathbf{E}_{\text {Bor }}\right)_{(0)}$. The natural transformation of equivariant cohomology theories appearing in (1.5) is compatible with the induced multiplicative structures.

In this discussion we are rather sloppy concerning the notion of a smash product. Since we are not dealing with higher structures and just want to take homotopy groups in the end, one can either use the classical approach in the sense of Adams or the more advanced new constructions such as symmetric spectra.

Lemma 5.2. The isomorphism appearing in Lemma 4.10 is compatible with the multiplicative structure on the source and the one on the target given by

$$
\left(a, u_{p,(C)}\right) \cdot\left(b, v_{p,(C)}\right)=\left(a \cdot b, a \cdot v_{p,(C)}+b \cdot v_{p,(C)}+u_{p,(C)} \cdot v_{p,(C)}\right),
$$

for

$$
\begin{aligned}
(C) & \in \mathcal{C}_{p}(G) \\
a, b & \in H^{*}(B G ; \mathbb{Q}) ; \\
u_{p,(C)}, v_{p,(C)} & \in H_{W_{G} C}^{*}\left(C_{G} C \backslash X^{C} ; \theta_{C} \cdot R(C) \otimes_{\mathbb{Z}} \mathbb{Q}_{p}\right),
\end{aligned}
$$

and the structures of a graded commutative ring on $\prod_{i \in \mathbb{Z}} H^{2 i+*}(B G ; \mathbb{Q})$ and $\prod_{i \in \mathbb{Z}} H_{W_{G} C}^{2 i+*}\left(C_{G} C \backslash X^{C} ; \theta_{C} \cdot R(C) \otimes_{\mathbb{Z}} \mathbb{Q}_{p}\right)$ coming from the cup-product and the multiplicative structure on $\theta_{C} \cdot R(C) \otimes_{\mathbb{Z}} \mathbb{Q}_{p}$ and the obvious $\prod_{i \in \mathbb{Z}} H^{2 i+*}(B G ; \mathbb{Q})$ module structure on $\prod_{i \in \mathbb{Z}} H_{W_{G} C}^{2 i+*}\left(C_{G} C \backslash X^{C} ; \theta_{C} \cdot R(C) \otimes_{\mathbb{Z}} \mathbb{Q}_{p}\right)$ coming from the canonical maps $C_{G} C \backslash X \rightarrow G \backslash X$ and $\mathbb{Q} \rightarrow \mathbb{Q}_{p}$.

Proof. The proof consists of a straightforward calculation which is essentially based on the following ingredients. In the sequel we use the notation of [20].

The equivariant Chern character of [20, Theorem 6.4] is compatible with the multiplicative structures.

In Theorem 0.3 we have analyzed for every finite group $H$ the multiplicative structure on

$$
K^{0}(B H) \cong \mathbb{Z} \times \prod_{p} \mathbb{I}_{p}(H) \otimes_{\mathbb{Z}} \mathbb{Z}_{p}
$$

Thus the Bredon cohomology group appearing in the target of the Chern character whose source is $H_{G}^{*}\left(X ;\left(\mathbf{K}_{\mathrm{Bor}}\right)_{(0)}\right)$ can be identified with

$$
\left(\prod_{i \in \mathbb{Z}} H^{*+2 i}(G \backslash X ; \mathbb{Q})\right) \times \prod_{p} \prod_{i \in \mathbb{Z}} H_{\mathbb{Q}_{p}^{\widehat{s}} \mathrm{Sub}(G ; \mathcal{F})}^{2 i+*}\left(X ; \mathbb{I}_{p}(?) \otimes_{\mathbb{Z}} \mathbb{Q}_{p}^{\widehat{p}}\right)
$$


with respect to the multiplicative structure analogously defined to the one appearing in Theorem 0.3 taking the obvious multiplicative structures on the factors and the module structures of the factor for $p$ over $\prod_{i \in \mathbb{Z}} H^{*+2 i}(G \backslash X ; \mathbb{Q})$ into account.

Fix a prime $p$. The $\mathbb{Q}_{p}[\operatorname{aut}(C)]$-map $R(C) \otimes_{\mathbb{Z}} \mathbb{Q}_{p} \rightarrow \theta_{C} \cdot R(C) \otimes_{\mathbb{Z}} \mathbb{Q}_{p}$ given by multiplication with the idempotent $\theta_{C}$ is compatible with the multiplicative structures. Using the identification of Lemma 4.9 we obtain for each cyclic $p$-group $C$ a retraction compatible with the multiplicative structures.

$$
\rho_{C}: \mathbb{I}_{p}(C) \otimes_{\mathbb{Z}} \mathbb{Q}_{p} \rightarrow T_{C}\left(\mathbb{I}_{p}(C) \otimes_{\mathbb{Z}} \mathbb{Q}_{p}\right)
$$

Recall that $T_{K}\left(\mathbb{I}_{p}(K) \otimes_{\mathbb{Z}} \mathbb{Q}_{p}\right)$ is trivial unless $K$ is a non-trivial cyclic $p$-group. Use these retractions as the maps $\rho_{K}$ in the definition of the isomorphism $\nu$ of $\mathbb{Q}_{p}^{\widehat{S}} \operatorname{Sub}(G ; \mathcal{F})$-modules for $M=\mathbb{I}_{p}(?) \otimes_{\mathbb{Z}} \mathbb{Q}_{p}^{\widehat{~}}$ in $[20,(5.1)]$. Then we obtain using the identification of Lemma 4.9 an isomorphism of $\mathbb{Q}_{p}^{\wedge} \mathrm{Sub}(G ; \mathcal{F})$-modules

$$
\mathbb{I}_{p}(?) \otimes_{\mathbb{Z}} \mathbb{Q}_{p} \stackrel{\cong}{\longrightarrow} \prod_{(C) \in \mathcal{C}_{p}} i(C) !\left(\theta_{C} \cdot R(C) \otimes_{\mathbb{Z}} \mathbb{Q}_{p}\right),
$$

which is compatible with the obvious multiplicative structure on the source and the one on the target given by the product of the multiplicative structures on the factors $i(C) !\left(\theta_{C} \cdot R(C) \otimes_{\mathbb{Z}} \mathbb{Q}_{p}\right)$ coming from the obvious one on $\theta_{C}$. $R(C) \otimes_{\mathbb{Z}} \mathbb{Q}_{p}$. Using the adjunction $\left(i(C)^{*}, i(C)_{!}\right)$this isomorphism induces an $\mathbb{Q} \hat{p}$-isomorphism compatible with the multiplicative structures

$$
H_{\mathbb{Q}_{p}^{r}}^{n} \operatorname{Sub}(G ; \mathcal{F})\left(X ; \mathbb{I}_{p}(?) \otimes_{\mathbb{Z}} \mathbb{Q}_{p}^{\widehat{p}}\right) \stackrel{\cong}{\rightrightarrows} \prod_{(C) \in \mathcal{C}_{p}(G)} H_{W_{G} C}^{n}\left(C_{G} C \backslash X ; \theta_{C} \cdot R(C) \otimes_{\mathbb{Z}} \mathbb{Q}_{p}^{\widehat{p}}\right) .
$$

Because the isomorphism in Lemma 4.11 (a) is compatible with the multiplicative structures, it implies together with Lemma 5.2

Lemma 5.3. For every proper $G$-CW-complex $X$ and $n \in \mathbb{Z}$ there is a $\mathbb{C}$ isomorphism, natural in $X$,

$$
\begin{aligned}
& \overline{\operatorname{ch}}_{G, \mathbb{C}}^{n}: H_{G}^{*}\left(X ;\left(\mathbf{K}_{\mathrm{Bor}}\right)_{(0)}\right) \otimes \mathbb{Q} \underset{\mathbb{C}}{\stackrel{\cong}{\rightarrow}} \\
& \left(\prod_{i \in \mathbb{Z}} H^{2 i+n}(G \backslash X ; \mathbb{C})\right) \times \prod_{p} \prod_{(g) \in \operatorname{con}_{p}(G)}\left(\prod_{i \in \mathbb{Z}} H^{2 i+n}\left(C_{G}\langle g\rangle \backslash X^{\langle g\rangle} ; \mathbb{Q}_{p} \otimes_{\mathbb{Q}} \mathbb{C}\right)\right),
\end{aligned}
$$

which is compatible with the multiplicative structure on the target given by

$$
\left(a, u_{p,(g)}\right) \cdot\left(b, v_{p,(g)}\right)=\left(a \cdot b,\left(a \cdot v_{p,(g)}+b \cdot u_{p,(g)}+u_{p,(g)} \cdot v_{p,(g)}\right)\right)
$$

for

$$
\begin{aligned}
(g) & \in \operatorname{con}_{p}(G) \\
a, b & \in \prod_{i \in \mathbb{Z}} H^{2 i+*}(G \backslash X ; \mathbb{C}), \\
u_{p,(g)}, v_{p,(g)} & \in \prod_{i \in \mathbb{Z}} H^{2 i+*}\left(C_{G}\langle g\rangle \backslash X^{\langle g\rangle} ; \mathbb{Q}_{p} \otimes_{\mathbb{Q}} \mathbb{C}\right),
\end{aligned}
$$


and the structures of a graded commutative ring on $\prod_{i \in \mathbb{Z}} H^{2 i+*}(G \backslash X ; \mathbb{C})$ and $\prod_{i \in \mathbb{Z}} H^{2 i+*}\left(C_{G}\langle g\rangle \backslash X^{\langle g\rangle} ; \mathbb{Q}_{p} \otimes_{\mathbb{Q}} \mathbb{C}\right)$ coming from the cup-product and the obvious $\prod_{i \in \mathbb{Z}} H^{2 i+*}(G \backslash X ; \mathbb{C})$-module structure on $\prod_{i \in \mathbb{Z}} H^{2 i+*}\left(C_{G}\langle g\rangle \backslash X^{\langle g\rangle} ; \mathbb{Q}_{p} \otimes_{\mathbb{Q}} \mathbb{C}\right)$ coming from the canonical map $B C_{G}\langle g\rangle \rightarrow B G$.

Now we are ready to prove Theorem 0.2

Proof. The isomorphism appearing in Lemma 1.6 is compatible with the multiplicative structures. This is also true for the versions of isomorphisms (4.15) and $(4.16)$, where the coefficients $\mathbb{Q}$ and $\mathbb{Q}_{p}$ are replaced by $\mathbb{C}$ and $\widehat{\mathbb{Q}_{p}} \otimes_{\mathbb{Q}} \mathbb{C}$. Now put these together with the isomorphism appearing in Lemma 5.3.

Remark 5.4. (Difference between rationalization and complexification). First of all we want to emphasize that the isomorphism appearing in Theorem 0.2 is not obtained from the isomorphism appearing in Theorem 0.1 by applying $-\otimes_{\mathbb{Q}} \mathbb{C}$ since the corresponding statement is already false for the two isomorphisms appearing in Lemma 4.11. Moreover, the isomorphism appearing in Theorem 0.1 is not compatible with the standard multiplicative structures on the source and the multiplicative structure on the target which is defined analogously to the one on the complexified target in Theorem 0.2 . The reason is that the isomorphism appearing in Lemma 4.11 (b) cannot be chosen to be compatible with the obvious multiplicative structure on its target if we use on the source the multiplicative structure coming from the obvious identification $\mathbb{Q}_{p}^{-}[\operatorname{Gen}(C)]=\prod_{\operatorname{Gen}(C)} \mathbb{Q}_{p}$ and the product ring structure on $\prod_{\operatorname{Gen}(C)} \mathbb{Q}_{p}$.

One can easily check by hand that there is no $\mathbb{Q} \widehat{3}[\operatorname{aut}(\mathbb{Z} / 3)]$-isomorphism compatible with the multiplicative structures

$$
\theta_{\mathbb{Z} / 3} \cdot R(\mathbb{Z} / 3) \otimes \mathbb{Q}_{\hat{3}}=\mathbb{I}_{\mathbb{Z} / 3} \otimes \mathbb{Q}_{3} \stackrel{=}{\rightarrow} \mathbb{Q}_{3} \times \mathbb{Q}_{3}
$$

if we equip the target with the $\operatorname{aut}(\mathbb{Z} / 3) \cong \mathbb{Z} / 2$-action given by flipping the factors and the product $\mathbb{Q}$-algebra structure. The point is that $\mathbb{Q}_{3}$ does not contain a primite 3 -rd root of unity (in contrast to $\mathbb{C}$, see Lemma 4.11 (a)).

Example 5.5 (Multiplicative structure over $\mathbb{Q}$ ). In general we can give a simple formula for the multiplicative structure only after complexifying as explained in Remark 5.4. In the following special case this can be done already after rationalization. Suppose that for any non-trivial cyclic subgroup $C$ of prime power order $\widetilde{H}^{n}\left(B C_{G} C ; \mathbb{Q}\right)=0$ holds for all $n \in \mathbb{Z}$ and that $W_{G} C=$ aut $(C)$. The latter means that any automorphism of $C$ is given by conjugation with some element in $N_{G} C$. Suppose furthermore that there is a finite model for $\underline{E} G$. Then we obtain $\mathbb{Q}$-isomorphisms

$$
\begin{aligned}
K^{0}(B G) \otimes_{\mathbb{Z}} \mathbb{Q} & \cong \prod_{i \in \mathbb{Z}} H^{2 i}(B G ; \mathbb{Q}) \times \prod_{p}\left(\widehat{\mathbb{Q}_{p}}\right)^{r_{p}(G)} ; \\
K^{1}(B G) \otimes_{\mathbb{Z}} \mathbb{Q} & \cong \prod_{i \in \mathbb{Z}} H^{2 i+1}(B G ; \mathbb{Q}),
\end{aligned}
$$


where $r_{p}(G)$ is the number of conjugacy classes of non-trivial cyclic subgroups of $p$-power order what is in this situation the same as $\left|\operatorname{con}_{p}(G)\right|$. The isomorphisms above are compatible with the multiplicative structure on the target given by

$$
\begin{aligned}
(a, u) \cdot(b, v) & =\left(a \cup b, a_{0} \cdot v+b_{0} \cdot u+u \cdot v\right) ; \\
(a, u) \cdot c & =a \cup c ; \\
c \cdot d & =c \cup d
\end{aligned}
$$

for $a, b \in \prod_{i \in \mathbb{Z}} H^{2 i}(B G ; \mathbb{Q}), c, d \in \prod_{i \in \mathbb{Z}} H^{2 i+1}(B G ; \mathbb{Q})$ and $u, v \in \prod_{p}\left(\mathbb{Q}_{p}^{-}\right)^{r_{p}(G)}$, where $a_{0} \in \mathbb{Q}$ and $b_{0} \in \mathbb{Q}$ are the components of $a$ and $b$ in $H^{0}(B G ; \mathbb{Q})=\mathbb{Q} \cdot 1$ and we equip $\prod_{p}\left(\mathbb{Q}_{p}^{-}\right)^{\left.r_{p}(G)\right)}$ with the structure of a $\mathbb{Q}$-algebra coming from the product of the obvious $\mathbb{Q}$-algebra structures on the various factors $\mathbb{Q}_{p}$. This follows from Lemma 5.2 and the conclusion from the formula $\theta_{C} \cdot \theta_{C}=\theta_{C}$ and Lemma 4.11 (b) that $\left(\theta_{C} \cdot R(C)\right)^{\text {aut }(C)}$ is generated as $\mathbb{Q}$-vector space by $\theta_{C}$ and hence is as $\mathbb{Q}$-algebra isomorphic to $\mathbb{Q}$.

If we furthermore assume that $\widetilde{H}_{n}(B G ; \mathbb{Q})=0$ for all $n \in \mathbb{Z}$, the formula simplifies to

$$
\begin{aligned}
K^{0}(B G) \otimes_{\mathbb{Z}} \mathbb{Q} & \cong \mathbb{Q} \times \prod_{p}\left(\mathbb{Q}_{p}^{-}\right)^{r_{p}(G)} ; \\
K^{1}(B G) \otimes_{\mathbb{Z}} \mathbb{Q} & \cong 0 .
\end{aligned}
$$

The first isomorphism is compatible with the multiplicative structures if we put on the target the one given by

$$
(m, a) \cdot(m, b)=(m n, m \cdot b+n \cdot a+a \cdot b)
$$

for $m, n \in \mathbb{Q}, a, b \in \prod_{p}\left(\mathbb{Q}_{p}\right)^{r_{p}(G)}$ and we equip $\prod_{p}\left(\mathbb{Q}_{p}\right)^{r_{p}(G)}$ with the structure of a $\mathbb{Q}$-algebra coming from the product of the obvious $\mathbb{Q}$-algebra structures on the various factors $\mathbb{Q} \widehat{p}$.

\section{Weakening the Finiteness Conditions}

In this section we want to weaken the finiteness assumption occurring in Theorem 0.1 and Theorem 0.2 .

A $\mathbb{Z}$-module $M$ is almost trivial if there is an element $r \in \mathbb{Z}, r \neq 0$ such that $r m=0$ holds for all $m \in M$. A $\mathbb{Z}$-module $M$ is almost finitely generated if $M / \operatorname{tors}(M)$ is a finitely generated $\mathbb{Z}$-module and tors $(M)$ is almost trivial. A $\mathbb{Z}$-homomorphism is an almost isomorphism if its kernel and cokernel are almost trivial. An almost isomorphism becomes an isomorphism after rationalization.

The full subcategories of the category of $\mathbb{Z}$-modules given by almost trivial submodules and by almost finitely generated submodules are Serre-subcategories, i.e. are closed under subobjects, quotients, and extensions. In particular there is 
a Five-Lemma for almost isomorphisms. These notions and facts are introduced and proved in [22, Section 4].

The main result of this section is:

Theorem 6.1 (Weakening the finiteness assumption). The conclusions of Theorem 0.1 and Theorem 0.2 remain true if we replace the condition that there is a cocompact $G-C W$-model for the classifying space $\underline{E} G$ for proper $G$-actions by the following weaker set of conditions:

There exists a $G-C W$-complex $X$ satisfying:

(a) The $G-C W$-complex $X$ is proper and finite dimensional. There is an upper bound on the orders of its isotropy groups. The set of conjugacy classes $(C)$ of finite cyclic subgroups $C \subseteq G$ of prime power order with $X^{C} \neq \emptyset$ is finite;

(b) For all for $k \in \mathbb{Z}$ we have $H_{k}(X ; \mathbb{Z}) \cong H_{k}(\{\bullet\} ; \mathbb{Z})$;

(c) For any finite cyclic subgroup of prime power order $C \subseteq G$ and integer $k$ the $\mathbb{Z}$-module $H_{k}\left(X^{C} ; \mathbb{Z}\right)$ is almost finitely generated;

(d) For any finite cyclic subgroup of prime power order $C \subseteq G$ and integer $k$ the $\mathbb{Z}$-module $H_{k}\left(C_{G} C \backslash X^{C} ; \mathbb{Z}\right)$ is almost finitely generated.

If $X$ satisfies conditions (a), (b) and (c) above, then the condition (d) is satisfied if and only if for any finite cyclic subgroup of prime power order $C \subseteq G$ and integer $k$ the $\mathbb{Z}$-module $H_{k}\left(B C_{G} C ; \mathbb{Z}\right)$ is almost finitely generated.

Remark 6.2 (Weakening the finiteness conditions for $\underline{E} G$ ). Notice that the conditions (a), (b), (c) and (d) in Theorem 6.1 are satisfied, if the set of conjugacy classes of finite subgroups of $G$ is finite, there is a finite dimensional model for $\underline{E} G$ and for any finite cyclic subgroup of prime power order $C \subseteq G$ and integer $k$ the $\mathbb{Z}$-module $H_{k}\left(B C_{G} C ; \mathbb{Z}\right)$ is almost finitely generated.

Remark 6.3 (Virtually torsionfree groups). Suppose that $G$ contains a torsionfree subgroup $H \subseteq G$ of finite index. If there is a finite dimensional model for $B H$, then there exists a finite dimensional model for $\underline{E} G$ [25]). However, if there is a finite model for $B H$, this does not implies that $G$ has only finitely many conjugacy classes of subgroups or that there is a cocompact model for $E G$ or that the centralizers $C_{G} C$ of finite cyclic subgroups are finitely generated [18, Section 7].

The proof of Theorem 6.1 needs some preparation.

Lemma 6.4. Let $X$ be a proper $G-C W$-complex. Let pr: $E G \times_{G} X \rightarrow G \backslash X$ be the projection. Fix an integer $n \in \mathbb{Z}$.

(a) Suppose that there exists for each $m \geq 0$ a positive integer $d(m)$ such that for any isotropy group $H$ of $X$ multiplication with $d(m)$ annihilates $\widetilde{H}_{m}(B H ; \mathbb{Z})$. Then the induced map

$$
H_{n}(\mathrm{pr} ; \mathbb{Z}): H_{n}\left(E G \times_{G} X ; \mathbb{Z}\right) \rightarrow H_{n}(G \backslash X ; \mathbb{Z}) .
$$

is an almost isomorphism for all $n \in \mathbb{Z}$; 
(b) The induced map

$$
H_{n}(\operatorname{pr} ; \mathbb{Q}): H_{n}\left(E G \times_{G} X ; \mathbb{Q}\right) \rightarrow H_{n}(G \backslash X ; \mathbb{Q}) .
$$

is a $\mathbb{Q}$-isomorphism.

Proof. (a) This is proved in [22, Lemma 8.1].

(b) The proof is analogous to the one of (b).

The next result is a generalization of Lemma 1.6 in the case $\mathbf{E}=\mathbf{K}$.

Lemma 6.5. Let $X$ be a finite dimensional proper $G-C W$-complex such that there is a bound on the orders of finite subgroups. Let $J$ be the set of conjugacy classes $(C)$ of finite cyclic subgroups $C \subseteq G$ of prime power order with $X^{C} \neq \emptyset$. Suppose that $|J|$ is finite. Furthermore assume that $H_{k}\left(C_{G} C \backslash X^{C} ; \mathbb{Z}\right)$ is almost finitely generated for every $k \in \mathbb{Z}$ and every finite cyclic subgroup of prime power order $C \subseteq G$.

Then the map

$$
i_{G}^{n}(X ; \mathbf{K}): H_{G}^{n}\left(X ; \mathbf{K}_{\mathrm{Bor}}\right) \otimes_{\mathbb{Z}} \mathbb{Q} \rightarrow H_{G}^{n}\left(X ;\left(\mathbf{K}_{\mathrm{Bor}}\right)_{(0)}\right)
$$

is bijective for all $n \in \mathbb{Z}$.

Proof. In the sequel we use the notation of [20]. Let $\mathcal{F}(X)$ be the set of conjugacy classes of subgroups of $H \subseteq G$ with $X^{H} \neq \emptyset$. Since $X$ is proper and has finite orbit type, $\mathcal{F}(X)$ is finite and $(H) \in \mathcal{F}(X)$ implies that $H$ is finite. Since $X$ is proper and finite dimensional, there is a spectral sequence converging to $H_{G}^{s+t}\left(X ; \mathbf{K}_{\text {Bor }}\right)$ whose $E_{2}$-term is $E_{2}^{s, t}=H_{\mathrm{Sub}(G ; \mathcal{F}(X))}^{s}\left(X ; K^{t}(B H)\right)$ and a spectral sequence converging to $H_{G}^{s+t}\left(X ;\left(\mathbf{K}_{\mathrm{Bor}}\right)_{(0)}\right)$ whose $E_{2}$-term is $E_{2}^{s, t}=$ $H_{\text {Sub }(G ; \mathcal{F}(X))}^{s}\left(X ; K^{t}(B H) \otimes_{\mathbb{Z}} \mathbb{Q}\right)$. Since $\mathbb{Q}$ is flat over $\mathbb{Z}$, it suffices to show that the canonical map

$$
H_{\mathrm{Sub}(G ; \mathcal{F}(X))}^{s}\left(X ; K^{t}(B H)\right) \otimes_{\mathbb{Z}} \mathbb{Q} \rightarrow H_{\mathrm{Sub}(G ; \mathcal{F}(X))}^{s}\left(X ; K^{t}(B H) \otimes_{\mathbb{Z}} \mathbb{Q}\right)
$$

is bijective for all $s$ and $t$. We have already explained that the contravariant $\mathbb{Z} \operatorname{Sub}(G ; \mathcal{F}(X))$-module sending $H$ to $K^{t}(B H)$ is zero for odd $t$ and given for even $t$ by

$$
K^{t}(B H) \cong \mathbb{Z} \times \prod_{p} \mathbb{I}_{p}(H) \otimes_{\mathbb{Z}} \mathbb{Z}_{p}^{\widehat{~}}
$$

One easily checks using Lemma 4.7 and (4.8) for any finite group $H$

$T_{H}\left(K^{0}(B H)\right) \cong \begin{cases}\mathbb{Z} & H=\{1\} ; \\ \operatorname{ker}\left(\operatorname{res}_{H}^{H^{\prime}}: R(H) \rightarrow R\left(H^{\prime}\right)\right) \otimes_{\mathbb{Z}} \mathbb{Z}_{p} & H \text { cyclic } p \text {-group, } \\ & H^{\prime} \subseteq H,\left[H: H^{\prime}\right]=p ; \\ 0 & \text { otherwise. }\end{cases}$

For every finite cyclic subgroup $K \subseteq G$ of order $p^{r}$ for some prime $p$ and integer $r \geq 1$ choose a retraction $r^{\prime}(K): K^{0}(B K) \rightarrow T_{K}\left(K^{0}(B K)\right)$ of the 
$\mathbb{Z}$-homomorphism $j(K): T_{K}\left(K^{0}(B K)\right) \rightarrow K^{0}(B K)$ given by inclusion. Such $r^{\prime}(K)$ exists since $R\left(K^{\prime}\right)$ and hence the image of $\operatorname{res}_{K}^{K^{\prime}}: R(K) \rightarrow R\left(K^{\prime}\right)$ is a finitely generated free $\mathbb{Z}$-module what implies that $\operatorname{ker}\left(\operatorname{res}_{K}^{K^{\prime}}: R(K) \rightarrow R\left(K^{\prime}\right)\right.$ ) is a direct summand of the finitely generated free $\mathbb{Z}$-module $\mathbb{I}_{p}(K)=\mathbb{I}(K)$. Since $W_{G} K$ is finite, we can define a $\mathbb{Z}\left[W_{G} K\right]$-map

$$
r(K): K^{0}(B K) \rightarrow T_{K}\left(K^{0}(B K)\right), \quad x \mapsto \sum_{g \in W_{G} K} g \cdot r^{\prime}(K)\left(g^{-1} \cdot x\right) .
$$

Then $r(K) \circ j(K)=\left|W_{G} K\right| \cdot$ id. For $K=\{1\}$ let $r(K): K^{0}(B K) \cong \mathbb{Z}$ be the obvious isomorphism which is for trivial reasons a $W_{G} K$-map. Define a map of contravariant $\operatorname{Sub}(G ; \mathcal{F}(X))$-modules

$$
\nu: K^{0}(B ?) \rightarrow \prod_{(K) \in J} i(K) ! T_{K}\left(K^{0}(B ?)\right)
$$

by requiring that the composite of $\nu$ with the projection onto the factor belonging to $(K) \in J$ is the adjoint for the pair $\left(i(K)^{*}, i(K) !\right)$ of the $W_{G} K$-map $r(K)$. Analogously to the proof of [20, Theorem $2.14(\mathrm{~b})]$ one shows that $\nu(H)$ is injective for all objects $H \in \operatorname{Sub}(G ; \mathcal{F}(X))$. Here we use the fact that $r(K) \circ j(K)$ is injective for all $K$ with $(K) \in J$ since $r(K) \circ j(K)=\left|W_{G} K\right| \cdot$ id and $K^{0}(B K)$ and hence $T_{K}\left(K^{0}(B K)\right)$ is torsionfree. Then one constructs analogously to the proof of $[20$, Theorem 5.2] for each object $(H) \in \operatorname{Sub}(G ; \mathcal{F}(X))$ a $\mathbb{Z}$-homomorphism

$$
\mu(H):\left(\prod_{(K) \in J} i(K) ! T_{K}\left(K^{0}(B ?)\right)\right)(H) \rightarrow K^{0}(B H)
$$

and checks that $\nu(H) \circ \mu(H)$ can be written as a diagonal matrix $A(H)$ which has upper triangular form and has maps of the shape $r$. id as diagional entry, where each $r$ divides a certain integer $M(|H|)$ depending only on the order of $|H|$. There is an integer $N(|H|)$ depending only on the order of $|H|$ such that the size of the square matrix $A(H)$ is bounded by $N(|H|)$. The existence of the numbers $M(|H|)$ and $N(|H|)$ follows from the finiteness of $J$. Hence for each object $H \in \operatorname{Sub}(G ; \mathcal{F}(X))$ the cokernel of $\nu(H)$ is annihilated by $M(|H|)^{N(|H|)}$. Since there is an upper bound on the orders of finite subgroups of $G$, we can find an integer $L$ such that for each object $H \in \operatorname{Sub}(G ; \mathcal{F}(X))$ the cokernel of $\nu(H)$ is annihilated by $L$. The short exact sequence of $\mathbb{Z} \operatorname{Sub}(G ; \mathcal{F}(X))$-modules

$$
0 \rightarrow K^{0}(B ?) \stackrel{\nu}{\rightarrow} \prod_{(K) \in J} i(K) ! T_{K}\left(K^{0}(B ?)\right) \stackrel{\mathrm{pr}}{\rightarrow} \operatorname{coker}(\nu) \rightarrow 0
$$

induces a long exact sequence

$$
\begin{aligned}
& \ldots \rightarrow H_{\operatorname{Sub}(G ; \mathcal{F}(X))}^{s-1}(X ; \operatorname{coker}(\nu)) \rightarrow H_{\operatorname{Sub}(G ; \mathcal{F}(X))}^{s}\left(X ; K^{0}(B ?)\right) \\
\rightarrow & H_{\text {Sub }(G ; \mathcal{F}(X))}^{s}\left(X ; \prod_{(K) \in J} i(K) ! T_{K}\left(K^{0}(B ?)\right)\right) \rightarrow H_{\text {Sub }(G ; \mathcal{F}(X))}^{s}(X ; \operatorname{coker}(\nu)) \rightarrow \ldots
\end{aligned}
$$


Since multiplication with $L$ induces the zero map $\operatorname{coker}(\nu) \rightarrow \operatorname{coker}(\nu)$, multiplication with $L$ induces also the zero map on $H_{\mathrm{Sub}(G ; \mathcal{F}(X))}^{s}(X ; \operatorname{coker}(\nu))$. Hence $H_{\mathrm{Sub}(G ; \mathcal{F}(X))}^{s}(X ; \operatorname{coker}(\nu)) \otimes_{\mathbb{Z}} \mathbb{Q}$ is trivial. Since $J$ is finite, and $-\otimes_{\mathbb{Z}} \mathbb{Q}$ is an exact functor which commutes with finite products, we obtain from the adjunction $\left(i(K)^{*} . i(K) !\right)$ a natural isomorphism

$$
H_{\mathrm{Sub}(G ; \mathcal{F}(X))}^{s}\left(X ; K^{0}(B ?)\right) \otimes_{\mathbb{Z}} \mathbb{Q} \stackrel{\cong}{\rightarrow} \prod_{(K) \in J} H_{W_{G} K}^{s}\left(C_{G} K \backslash X^{K} ; T_{K} K^{0}(B ?)\right) \otimes_{\mathbb{Z}} \mathbb{Q} .
$$

Similarly we get an isomorphism

$$
H_{\mathrm{Sub}(G ; \mathcal{F}(X))}^{s}\left(X ; K^{0}(B ?) \otimes_{\mathbb{Z}} \mathbb{Q}\right) \stackrel{\cong}{\rightarrow} \prod_{(K) \in J} H_{W_{G} K}^{s}\left(C_{G} K \backslash X^{K} ; T_{K} K^{0}(B ?) \otimes_{\mathbb{Z}} \mathbb{Q}\right) .
$$

Hence it remains to show for each $(K) \in J$ and $s \geq 0$ that the canonical map

$$
H_{W_{G} K}^{s}\left(C_{G} K \backslash X^{K} ; T_{K} K^{0}(B ?)\right) \otimes_{\mathbb{Z}} \mathbb{Q} \rightarrow H_{W_{G} K}^{s}\left(C_{G} K \backslash X^{K} ; T_{K} K^{0}(B ?) \otimes_{\mathbb{Z}} \mathbb{Q}\right)
$$

is bijective. Abbreviate $C_{*}=C_{*}\left(C_{G} K \backslash Y^{K}\right), L=W_{G} K$ and $M=T_{K} K^{0}(B$ ?). Then $L$ is a finite group, $C_{*}$ is a $\mathbb{Z} L$-chain complex which is free over $\mathbb{Z}$ and for which there exists an integer $n \geq 1$ such that tors $\left(H_{s}\left(C_{*}\right)\right)$ is annihilated by $n$ and $H_{s}\left(C_{*}\right) / \operatorname{tors}\left(H_{s}\left(C_{*}\right)\right)$ is a finitely generated $\mathbb{Z}$-module for all $s$. It remains to show for the $\mathbb{Z} L$-module $M$ that the canonical map

$$
H^{s}\left(\operatorname{hom}_{\mathbb{Z} L}\left(C_{*}, M\right)\right) \otimes_{\mathbb{Z}} \mathbb{Q} \rightarrow H^{s}\left(\operatorname{hom}_{\mathbb{Z} L}\left(C_{*}, M \otimes_{\mathbb{Z}} \mathbb{Q}\right)\right)
$$

is bijective for all $s$. Let $i:\{1\} \rightarrow L$ be the inclusion. Since $L$ is finite, induction $i_{*}$ and coinduction $i_{\text {! }}$ agree. Hence we get natural $\mathbb{Z} L$-chain maps $a_{*}: C_{*} \rightarrow$ $i_{*} i^{*} C_{*}$ and $b_{*}: i_{*} i^{*} C_{*} \rightarrow C_{*}$ such that $b_{*} \circ a_{*}$ is multiplication with $|L|$. They are explicitly given by

$$
\begin{array}{ll}
a_{s}: C_{s} \rightarrow \mathbb{Z} L \otimes_{\mathbb{Z}} C_{s}, & x \mapsto \sum_{l \in L} l \otimes l^{-1} \cdot x ; \\
b_{s}: \mathbb{Z} L \otimes_{\mathbb{Z}} C_{s} \rightarrow C_{s}, & l \otimes y \mapsto l \cdot y .
\end{array}
$$

Hence we obtain a commutative diagram

$$
\begin{array}{ccc}
H^{s}\left(\operatorname{hom}_{\mathbb{Z} L}\left(C_{*}, M\right)\right) \otimes_{\mathbb{Z}} \mathbb{Q} & H^{s}\left(\operatorname{hom}_{\mathbb{Z} L}\left(C_{*}, M \otimes_{\mathbb{Z}} \mathbb{Q}\right)\right) \\
H^{s}\left(b_{*}\right) \otimes_{\mathbb{Z}} \text { id } & \\
H^{s}\left(b_{*}\right) \downarrow & H^{s}\left(\operatorname{hom}_{\mathbb{Z} L}\left(\mathbb{Z} L \otimes_{\mathbb{Z}} C_{*}, M \otimes_{\mathbb{Z}} \mathbb{Q}\right)\right) \\
H^{s}\left(a_{*}\right) \otimes_{\mathbb{Z}} \otimes_{\mathbb{Z}} \downarrow & \left.\left.C_{*}, M\right)\right) \otimes_{\mathbb{Z}} \mathbb{Q} & H^{s}\left(a_{*}\right) \downarrow \\
H^{s}\left(\operatorname{hom}_{\mathbb{Z} L}\left(C_{*}, M\right)\right) \otimes_{\mathbb{Z}} \mathbb{Q} & \longrightarrow & H^{s}\left(\operatorname{hom}_{\mathbb{Z} L}\left(C_{*}, M \otimes_{\mathbb{Z}} \mathbb{Q}\right)\right)
\end{array}
$$

where the horizontal arrows are the canonical maps and the composite of the two left vertical maps and the composite of the two right vertical maps are 
isomorphisms. Hence it suffices to show that the middle horizontal arrow is an isomorphism. It can be identified with the canonical map

$$
H^{s}\left(\operatorname{hom}_{\mathbb{Z}}\left(C_{*}, M\right)\right) \otimes_{\mathbb{Z}} \mathbb{Q} \rightarrow H^{s}\left(\operatorname{hom}_{\mathbb{Z}}\left(C_{*}, M \otimes_{\mathbb{Z}} \mathbb{Q}\right)\right) .
$$

Notice for the sequel that $C_{*}$ is $\mathbb{Z}$-free. By the universal coefficient theorem we get a commutative diagram with exact rows and the canonical maps as horizontal arrows

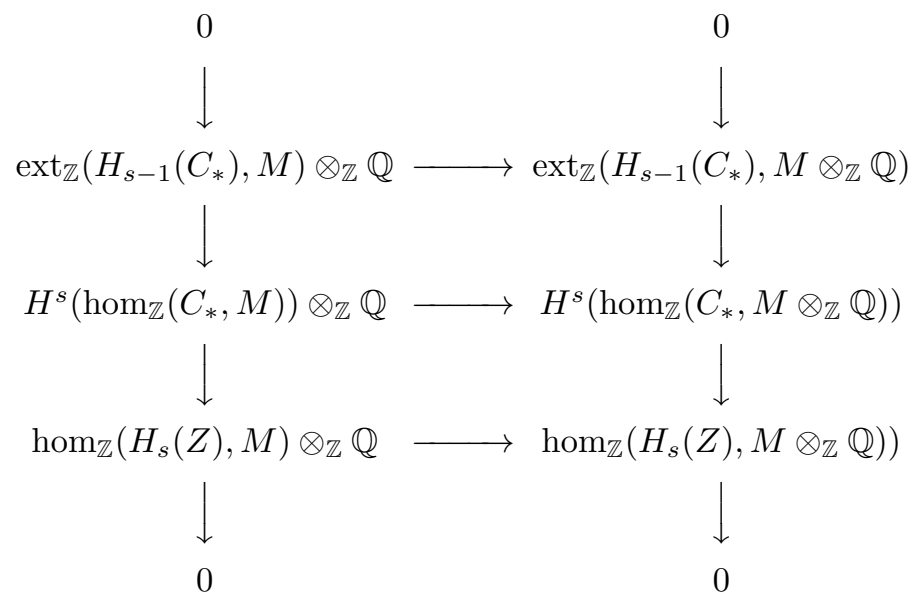

Since $H_{s}\left(C_{*}\right) / \operatorname{tors}\left(H_{s}\left(C_{*}\right)\right.$ is a finitely generated free abelian group and there is an integer $n$ which annihilates tors $\left(H_{s}\left(C_{*}\right)\right)$, the rational vector spaces $\operatorname{ext}_{\mathbb{Z}}\left(H_{s-1}\left(C_{*}\right), M\right) \otimes_{\mathbb{Z}} \mathbb{Q}$ and $\operatorname{ext}_{\mathbb{Z}}\left(H_{s-1}\left(C_{*}\right), M \otimes_{\mathbb{Z}} \mathbb{Q}\right)$ vanish and the lower vertical arrow is bijective. Hence the middle arrow is bijective. This finishes the proof of Lemma 6.5.

Lemma 6.6. Let $X$ be a proper $G-C W$-complex such that for any cyclic subgroup $C \subseteq G$ of prime power order and any $k \in \mathbb{Z}$ we have $H_{k}\left(X^{C} ; \mathbb{Q}\right) \cong$ $H_{k}(\{\bullet\} ; \mathbb{Q})$. Then the up to $G$-homotopy unique $G$-map $f: X \rightarrow \underline{E} G$ induces for every $n \in \mathbb{Z}$ an isomorphism

$$
H_{G}^{n}\left(f ;\left(\mathbf{K}_{\mathrm{Bor}}\right)_{(0)}\right): H_{G}^{n}\left(\underline{E} G ;\left(\mathbf{K}_{\mathrm{Bor}}\right)_{(0)}\right) \stackrel{\cong}{\rightrightarrows} H_{G}^{n}\left(X ;\left(\mathbf{K}_{\mathrm{Bor}}\right)_{(0)}\right) .
$$

Proof. Because of Lemma 4.12 it suffices to show for every $n \in \mathbb{Z}$ and every cyclic subgroup of prime power order that the map

$$
H^{n}\left(C_{G} C \backslash f^{C} ; M\right): H^{n}\left(C_{G} C \backslash(\underline{E} G)^{C} ; M\right) \rightarrow H^{n}\left(C_{G} C \backslash X^{C} ; M\right)
$$

is bijective for any $\mathbb{Q}$-module $M$. The Atiyah-Hirzebruch spectral sequence for the fibration $X^{C} \rightarrow E C_{G} C \times_{C_{G} C} X^{C} \rightarrow B C_{G} C$ together with the vanishing of $\widetilde{H}_{*}\left(X^{C} ; \mathbb{Q}\right)$ implies that the projection pr: $E C_{G} C \times_{C_{G} C} X^{C} \rightarrow B C_{G} C$ induces for all $n \in \mathbb{Z}$ isomorphisms

$$
H_{n}(\operatorname{pr} ; \mathbb{Q}): H_{n}\left(E C_{G} C \times_{C_{G} C} X^{C} ; \mathbb{Q}\right) \rightarrow H_{n}\left(B C_{G} C ; \mathbb{Q}\right) .
$$


The projection $\mathrm{pr}^{\prime}: E C_{G} C \times_{C_{G} C} X^{C} \rightarrow C_{G} C \backslash X^{C}$ induces for all $n \in \mathbb{Z}$ isomorphisms

$$
H_{n}\left(\mathrm{pr}^{\prime} ; \mathbb{Q}\right): H_{n}\left(E C_{G} C \times{ }_{C_{G} C} X^{C} ; \mathbb{Q}\right) \rightarrow H_{n}\left(C_{G} C \backslash X^{C} ; \mathbb{Q}\right)
$$

by Lemma 6.4 (b). The same is also true for $\underline{E} G$ instead of $X$. This implies that

$$
H_{n}\left(C_{G} C \backslash f^{C} ; \mathbb{Q}\right): H_{n}\left(C_{G} C \backslash X^{C} ; \mathbb{Q}\right) \rightarrow H_{n}\left(C_{G} C \backslash(\underline{E} G)^{C} ; \mathbb{Q}\right)
$$

is bijective for all $n \in \mathbb{Z}$. Hence $H^{n}\left(C_{G} C \backslash f^{C} ; M\right)$ is bijective for any $\mathbb{Q}$-module $M$.

Lemma 6.7. Let $X$ be a proper finite dimensional $G-C W$-complex such that $H_{k}(X ; \mathbb{Z}) \cong H_{k}(\{\bullet\} ; \mathbb{Z})$ holds for any $k \in \mathbb{Z}$. Let $C \subseteq G$ be a cyclic group of prime power order. Suppose that $\widetilde{H}_{n}\left(X^{C} ; \mathbb{Z}\right)$ is almost finitely generated for each $n \in \mathbb{Z}$. Then:

(a) The $\mathbb{Z}$-module $\widetilde{H}_{n}\left(X^{C} ; \mathbb{Z}\right)$ is almost trivial and the $\mathbb{Q}$-module $\widetilde{H}_{n}\left(X^{C} ; \mathbb{Q}\right)$ is trivial for all $n \in \mathbb{Z}$;

(b) The map $H_{n}(\mathrm{pr} ; \mathbb{Z}): H_{n}\left(E C_{G} C \times_{C_{G} C} X^{C} ; \mathbb{Z}\right) \rightarrow H_{n}\left(B C_{G} C ; \mathbb{Z}\right)$ induced by the projection pr: $E C_{G} C \times{ }_{C_{G} C} X^{C} \rightarrow B C_{G} C$ is an almost isomorphism for all $n \in \mathbb{Z}$.

Proof. (a) Suppose that $C$ has order $p^{k}$ for $k \geq 1$. Then $\widetilde{H}_{n}\left(X ; \mathbb{F}_{p}\right)=0$ for all $n \in \mathbb{Z}$ if $\mathbb{F}_{p}$ is the finite field of order $p$. By Smith theory $\widetilde{H}_{n}\left(X^{C} ; \mathbb{F}_{p}\right)=0$ for all $n \in \mathbb{Z}$ [9, Theorem 5.2]. This implies by the Bockstein sequence associated to $0 \rightarrow \mathbb{Z} \stackrel{p \text {.id }}{\longrightarrow} \mathbb{Z} \rightarrow \mathbb{F}_{p} \rightarrow 0$ that $p \cdot$ id: $\widetilde{H}_{n}\left(X^{C} ; \mathbb{Z}\right) \rightarrow \widetilde{H}_{n}\left(X^{C} ; \mathbb{Z}\right)$ is bijective for $n \in \mathbb{Z}$. Since $\widetilde{H}_{n}\left(X^{C} ; \mathbb{Z}\right)$ is almost finitely generated, it must be almost trivial for $n \geq 1$. This implies that $\widetilde{H}_{n}\left(X^{C} ; \mathbb{Q}\right)=0$ for all $n \in \mathbb{Z}$.

(b) This follows from the Lerray-Serre spectral sequence of the fibration $X^{C} \rightarrow$ $E C_{G} C \times X^{C} \rightarrow B C_{G} C$ whose $E^{2}$-term is $H_{s}\left(B C_{G} C ; \widetilde{H}_{t}\left(X^{C} ; \mathbb{Z}\right)\right)$ and which converges to $H_{s+t}$ (pr: $\left.E C_{G} C \times_{C_{G} C} X^{C} \rightarrow B C_{G} C ; \mathbb{Z}\right)$ and the fact that the full subcategory of almost trivial $\mathbb{Z}$-modules is a Serre subcategory of the abelian category of $\mathbb{Z}$-modules.

Now we can give the proof of Theorem 6.1.

Proof. If one goes through the proofs of Theorem 0.1 and Theorem 0.2 one sees that the finiteness condition about $\underline{E} G$ enters only, when we apply Lemma 1.6 to $\underline{E} G$. Hence it suffices to show that under the assumptions appearing in Theorem 6.1 the map

$$
i_{G}^{n}(\underline{E} G ; \mathbf{K}): H_{G}^{n}\left(\underline{E} G ; \mathbf{K}_{\mathrm{Bor}}\right) \otimes_{\mathbb{Z}} \mathbb{Q} \rightarrow H_{G}^{n}\left(\underline{E} G ;\left(\mathbf{K}_{\mathrm{Bor}}\right)_{(0)}\right)
$$

is a $\mathbb{Q}$-isomorphism for all $n \in \mathbb{Z}$.

Let $f: X \rightarrow \underline{E} G$ be the up to $G$-homotopy unique $G$-map. We obtain the following commutative diagram 


$$
\begin{array}{cc}
H_{G}^{n}\left(\underline{E} G ; \mathbf{K}_{\mathrm{Bor}}\right) \otimes_{\mathbb{Z}} \mathbb{Q} \stackrel{i_{G}^{n}(\underline{E} G ; \mathbf{K})}{\longrightarrow} H_{G}^{n}\left(\underline{E} G ;\left(\mathbf{K}_{\mathrm{Bor}}\right)_{(0)}\right) \\
H_{G}^{n}\left(f ; \mathbf{K}_{\mathrm{Bor}}\right) \otimes_{\mathbb{Z}} \downarrow \\
H_{G}^{n}\left(X ; \mathbf{K}_{\mathrm{Bor}}\right) \otimes_{\mathbb{Z}} \mathbb{Q}\left(\underline{E} G ;\left(\mathbf{K}_{\mathrm{Bor}}\right)_{(0)}\right) \\
\stackrel{ }{i_{G}^{n}(X ; \mathbf{K})} & H_{G}^{n}\left(X ;\left(\mathbf{K}_{\mathrm{Bor}}\right)_{(0)}\right)
\end{array}
$$

Since $H_{k}(f, \mathbb{Z}): H_{k}(X ; \mathbb{Z}) \rightarrow H_{k}(\underline{E} G ; \mathbb{Z})$ is bijective for all $k \in \mathbb{Z}$, we conclude from the Lerray-Serre spectral sequence that $H_{k}\left(E G \times_{G} f, \mathbb{Z}\right): H_{k}\left(\left(E G \times_{G}\right.\right.$ $X ; \mathbb{Z}) \rightarrow H_{k}\left(\left(E G \times_{G} \underline{E} G ; \mathbb{Z}\right)\right.$ is bijective for all $k \in \mathbb{Z}$. This implies that the left vertical arrow in the commutative square above which can be identified with $K^{n}\left(E G \times_{G} f\right): K_{n}\left(E G \times_{G} \underline{E} G\right) \rightarrow K_{n}\left(E G \times_{G} X\right)$ is bijective. The lower horizontal arrow is bijective by Lemma 6.5 . The right vertical arrow is bijective by Lemma 6.6 and Lemma 6.7 (a). Hence the upper horizontal arrow is bijective.

The claim about the equivalent reformulation of condition (d) follows from Lemma 6.4 (a) and Lemma 6.7 (b). This finishes the proof of Theorem 6.1.

\section{Examples and Further Remarks}

Some finiteness conditions such as appearing in Theorem 6.1 are necessary as the following example shows.

Example 7.1. (Necessity of the finiteness conditions). Consider $G=$ $*_{i=1}^{\infty} \mathbb{Z} / p$ for a prime number $p$. Then $B G \simeq \bigvee_{i=1}^{\infty} B \mathbb{Z} / p$ and we get

$$
K^{0}(B G) \cong K^{0}(\{\bullet\}) \times \prod_{i=1}^{\infty} \widetilde{K}^{0}(B \mathbb{Z} / p) \cong \mathbb{Z} \times \prod_{i=1}^{\infty}\left(\mathbb{Z}_{p}^{\widehat{p}}\right)^{p-1}
$$

if $\{\bullet\}$ is the one-point-space. Since $H^{n}(B G ; M) \cong \prod_{i=1}^{\infty} H^{n}(B \mathbb{Z} / p ; M)=0$ for any $\mathbb{Q}$-module $M$ and $n \geq 2$, the cohomological dimension of $G$ over $\mathbb{Q}$ is $\leq 1$ and hence $G$ acts on a tree $T$ with finite stabilizers [13]. Then $T$ is a 1dimensional model for $\underline{E} G$ (see [27, page 20] or [12, Proposition 4.7 on page 17]). By the Kurosh Subgroup Theorem [24, Theorem 1.10 on page 178]) any nontrivial finite subgroup of $G$ is conjugated to precisely one of the summands $\mathbb{Z} / p$ and is equal to its centralizer. Hence $p$ is an upper bound on the orders of finite subgroups of $G$. Obviously $\mathbb{Z}_{p} \otimes_{\mathbb{Z}} \mathbb{Q}$ is canonically isomorphic to $\mathbb{Q}_{\hat{p}}$. If the conclusion of Theorem 0.1 would be true for $G$, it would predict that the canonical map

$$
\left(\prod_{i=1}^{\infty}\left(\mathbb{Z}_{p}\right)^{p-1}\right) \otimes_{\mathbb{Z}} \mathbb{Q} \rightarrow \prod_{i=1}^{\infty}\left(\left(\mathbb{Z}_{p}^{\widehat{p}}\right)^{p-1} \otimes_{\mathbb{Z}} \mathbb{Q}\right)=\prod_{i=1}^{\infty}\left(\mathbb{Q}_{p}^{\widehat{p}}\right)^{p-1}
$$

is bijective, what is not true. For instance, the element $\left(p^{-i}\right)_{i=1}^{\infty}$ is not contained in its image. 
Notice that in this example all conditions appearing in Theorem 6.1 are satisfied except the condition that the set of conjugacy classes $(C)$ of finite cyclic subgroups $C \subseteq G$ of prime power order with $T^{C} \neq \emptyset$ is finite;

We emphasize that no restriction (except properness) occur in Lemma 4.12. The problem is in Lemma 6.6 some additional finiteness assumptions are needed.

Example 7.2 $\left(S L_{3}(\mathbb{Z})\right)$. Consider the group $G=S L_{3}(\mathbb{Z})$. It is well-known that its rational cohomology satisfies $\widetilde{H}^{n}\left(B S L_{3}(\mathbb{Z}) ; \mathbb{Q}\right)=0$ for all $n \in \mathbb{Z}$. Actually, we conclude from $\left[28\right.$, Corollary on page 8] that for $G=S L_{3}(\mathbb{Z})$ the quotient space $G \backslash \underline{E} G$ is contractible and compact. From the classification of finite subgroups of $S L_{3}(\mathbb{Z})$ we see that $S L_{3}(\mathbb{Z})$ contains up to conjugacy two elements of order 2, two elements of order 4 and two elements of order 3 and no further conjugacy classes of non-trivial elements of prime power order. The rational homology of all the centralizers of elements in $\operatorname{con}_{2}(G)$ and $\operatorname{con}_{3}(G)$ agree with the one of the trivial group (see [2, Example 6.6]). Hence Theorem 0.1 shows

$$
\begin{aligned}
& K^{0}\left(B S L_{3}(\mathbb{Z})\right) \otimes_{\mathbb{Z}} \mathbb{Q} \cong \mathbb{Q} \times\left(\mathbb{Q}_{2}\right)^{4} \times\left(\mathbb{Q}_{3}\right)^{2} ; \\
& K^{1}\left(B S L_{3}(\mathbb{Z})\right) \otimes_{\mathbb{Z}} \mathbb{Q} \cong 0 .
\end{aligned}
$$

The identification of $K^{0}\left(B S L_{3}(\mathbb{Z})\right) \otimes_{\mathbb{Z}} \mathbb{Q}$ above is compatible with the multiplicative structure on the target described in Example 5.5.

Actually the computation using Brown-Petersen cohomology and the ConnerFloyd relation by Tezuka and Yagita [30] gives the integral computation

$$
\begin{aligned}
& K^{0}\left(B S L_{3}(\mathbb{Z})\right) \cong \mathbb{Z} \times\left(\mathbb{Z}_{2}\right)^{4} \times\left(\mathbb{Z}_{3}\right)^{2} ; \\
& K^{1}\left(B S L_{3}(\mathbb{Z})\right) \cong 0 \text {. }
\end{aligned}
$$

Example 7.3 (Groups with appropriate maximal finite subgroups). Let $G$ be a discrete group. Consider the following assertions concerning $G$ :

(M) Every non-trivial finite subgroup of $G$ is contained in a unique maximal finite subgroup;

(NM) If $M \subseteq G$ is maximal finite, then $N_{G} M=M$;

(C) There is a cocompact model for $\underline{E} G$.

The conditions (M) and (NM) imply the following: For any non-trivial finite subgroup $H \subseteq G$ we have $N_{G} H=N_{M} H$ if $M$ is a maximal finite subgroup containing $H$. Let $\left\{M_{i} \mid i \in I\right\}$ be a complete set of representatives of the conjugacy classes of maximal finite subgroups of $G$. Fix a prime $p$. Then the obvious map

$$
\coprod_{i \in I} \operatorname{con}_{p}\left(M_{i}\right) \stackrel{\cong}{\rightrightarrows} \operatorname{con}_{p}(G)
$$

is a bijection. Let $r_{p}\left(M_{i}\right)=\left|\operatorname{con}_{p}\left(M_{i}\right)\right|$ be the number of conjugacy classes of elements in $M_{i}$ of order $p^{k}$ for some $k \geq 1$. 
Theorem 0.1 yields for a group satisfying conditions (M), (NM) and (C) above rational isomorphisms

$$
\begin{aligned}
K^{0}(B G) \otimes_{\mathbb{Z}} \mathbb{Q} & \cong \prod_{i \in \mathbb{Z}} H^{2 i}(B G ; \mathbb{Q}) \times \prod_{p}\left(\widehat{\mathbb{Q}_{p}}\right)^{\sum_{i \in I} r_{p}\left(M_{i}\right)} \\
K^{1}(B G) \otimes_{\mathbb{Z}} \mathbb{Q} & \cong \prod_{i \in \mathbb{Z}} H^{2 i+1}(B G ; \mathbb{Q}) .
\end{aligned}
$$

Here are some examples of groups $Q$ which satisfy conditions (M), (NM) and (C)

- Extensions $1 \rightarrow \mathbb{Z}^{n} \rightarrow G \rightarrow F \rightarrow 1$ for finite $F$ such that the conjugation action of $F$ on $\mathbb{Z}^{n}$ is free outside $0 \in \mathbb{Z}^{n}$.

The conditions (M), (NM) are satisfied by [23, Lemma 6.3]. There are models for $\underline{E} G$ whose underlying space is $\mathbb{R}^{n}$. The quotient $G \backslash \underline{E} G$ looks like the quotient of $T^{n}$ by a finite group.

- Fuchsian groups $F$

See for instance [23, Lemma 4.5]). The quotients $G \backslash \underline{E} G$ are closed orientable surfaces. In [23] the larger class of cocompact planar groups (sometimes also called cocompact NEC-groups) is treated.

- Finitely generated one-relator groups $G$

Let $G=\left\langle\left(q_{i}\right)_{i \in I} \mid r\right\rangle$ be a presentation with one relation. We only have to consider the case, where $Q$ contains torsion. Let $F$ be the free group with basis $\left\{q_{i} \mid i \in I\right\}$. Then $r$ is an element in $F$. There exists an element $s \in F$ and an integer $m \geq 2$ such that $r=s^{m}$, the cyclic subgroup $C$ generated by the class $\bar{s} \in Q$ represented by $s$ has order $m$, any finite subgroup of $G$ is subconjugated to $C$ and for any $q \in Q$ the implication $q^{-1} C q \cap C \neq$ $1 \Rightarrow q \in C$ holds. These claims follows from [24, Propositions 5.17, 5.18 and 5.19 in II.5 on pages 107 and 108]. Hence $Q$ satisfies (M) and $(\mathrm{NM})$. There are explicit two-dimensional models for $\underline{E} G$ with one 0-cell $G / C \times D^{0}$, as many free 1-cells $G \times D^{1}$ as there are elements in $I$ and one free 2-cell $G \times D^{2}$ (see [10, Exercise 2 (c) II. 5 on page 44]).

For the three examples above one can make $H^{*}(B G ; \mathbb{Q})=H^{*}(G \backslash \underline{E} G ; \mathbb{Q})$ more explicit.

Example 7.4 (Extensions of $\mathbb{Z}^{n}$ with $\mathbb{Z} / p$ as quotient). Suppose that $G$ can be written as an extension $1 \rightarrow A \rightarrow G \rightarrow \mathbb{Z} / p \rightarrow 1$ for some fixed prime number $p$ and for $A=\mathbb{Z}^{n}$ for some integer $n \geq 0$ and that $G$ is not torsionfree. The conjugation action of $G$ on the normal subgroup $A$ yields the structure of a $\mathbb{Z}[\mathbb{Z} / p]$-module on $A$. Every non-trivial element $g \in G$ of finite order $G$ has order $p$ and satisfies

$$
N_{G}\langle g\rangle=C_{G}\langle g\rangle=A^{\mathbb{Z} / p} \times\langle g\rangle .
$$

There is a bijection

$$
\mu: H^{1}(\mathbb{Z} / p ; A) \times(\mathbb{Z} / p)^{\times} \stackrel{\cong}{\rightrightarrows} \operatorname{con}_{p}(G),
$$


where $H^{1}(\mathbb{Z} / p ; A)$ is the first cohomology of $\mathbb{Z} / p$ with coefficients in the $\mathbb{Z}[\mathbb{Z} / p]$ module $A$. If we fix an element $g \in G$ of order $p$ and a generator $s \in \mathbb{Z} / p$, the bijection $\mu$ sends $([u], \bar{k}) \in H^{1}(\mathbb{Z} / p ; A) \times(\mathbb{Z} / p)^{\times}$to the conjugacy class $\left(u g^{k}\right)$ of $a g^{k}$ if $[u] \in H^{1}(\mathbb{Z} / p ; A)$ is represented by the element $u$ in the kernel of the second differential $A \rightarrow A, a \mapsto \sum_{i=0}^{p-1} s^{i} \cdot a$ and $k \in \mathbb{Z}$ represents $\bar{k}$. There is a cocompact model for $\underline{E} G$ with $A \otimes_{\mathbb{Z}} \mathbb{R}$ as underlying space. Hence Theorem 0.1 yields for $G$ as above rational isomorphisms

$$
K^{n}(B G) \otimes_{\mathbb{Z}} \mathbb{Q} \cong \prod_{i \in \mathbb{Z}} H^{2 i+n}(B A ; \mathbb{Q})^{\mathbb{Z} / p} \times \prod_{k=1}^{r} \prod_{i \in \mathbb{Z}} H^{2 i+n}\left(B\left(A^{\mathbb{Z} / p}\right) ; \mathbb{Q}_{p}^{r}\right),
$$

if we put $r=(p-1) \cdot\left|H^{1}(\mathbb{Z} / p ; A)\right|$.

Take for instance $A$ to be the cokernel of the inclusion of $\mathbb{Z}[\mathbb{Z} / p]$-modules $\mathbb{Z} \rightarrow \mathbb{Z}[\mathbb{Z} / p], n \mapsto n \cdot \sum_{i=0}^{p-1} t^{i}$, where $\mathbb{Z}$ carries the trivial $\mathbb{Z} / p$-action and $t \in \mathbb{Z} / p$ is a fixed generator. One can identify $A$ with the extension of $\mathbb{Z}$ by adjoining a primitive $p$-th root of unity. From the long exact cohomology sequence associated to the short exact sequence of $\mathbb{Z}[\mathbb{Z} / p]$-modules $0 \rightarrow \mathbb{Z} \rightarrow \mathbb{Z}[\mathbb{Z} / p] \rightarrow A \rightarrow 0$ one concludes that $H^{1}(\mathbb{Z} / p ; A)$ is a cyclic group of order $p$. One easily checks that $A^{\mathbb{Z} / p}=0$. Hence we obtain for the semi-direct product $A \rtimes \mathbb{Z} / p$

$$
\begin{aligned}
& K^{0}(B(A \rtimes \mathbb{Z} / p)) \otimes_{\mathbb{Z}} \mathbb{Q} \cong \mathbb{Q} \times\left(\mathbb{Q}_{p}\right)^{p^{2}-p} ; \\
& K^{1}(B(A \rtimes \mathbb{Z} / p)) \otimes_{\mathbb{Z}} \mathbb{Q} \cong 0 .
\end{aligned}
$$

The identification of $K^{0}(B(A \rtimes \mathbb{Z} / p)) \otimes_{\mathbb{Z}} \mathbb{Q}$ is compatible with the multiplicative structure on the target described in Example 5.5.

Remark 7.5 (Comparison with Adem's work). The results and the examples appearing in this paper are consistent with the ones by Adem [2]. Adem needs that $G$ contains a normal torsionfree subgroup $G^{\prime} \subseteq G$ of finite index and uses the Atiyah-Segal Completion Theorem for the finite group $G / G^{\prime}$ to compute rationally the $K$-theory with coefficients in the $p$-adic integers $\mathbb{Z}_{p}^{\widehat{~}}$. His condition that in his notation $\Gamma^{\prime} \backslash X$ is compact is precisely the condition that there is a cocompact model for $\underline{E} G$. We can drop the condition of the existence of the normal torsionfree subgroup $G^{\prime} \subseteq G$ of finite index with our methods.

One can get Adem's local computations from ours by replacing for a fixed prime $p$ the cohomology $H^{*}(B G ; \mathbb{Q})$ by $H^{*}\left(B G ; \mathbb{Q}_{p}\right)$ and ignoring in the product running over all primes all the contributions coming from primes different from $p$. For instance Example 7.2 implies that the $\mathbb{Q}_{\hat{3}}$-algebra $K^{0}\left(B S L(3, \mathbb{Z}) ; \mathbb{Z}_{3}\right) \otimes_{\mathbb{Z} \hat{3}} \mathbb{Q}_{3}$ is given by

$$
\mathbb{Q}_{3}[u, v] /\left(u^{2}=u, v^{2}=v, u v=0\right) .
$$

If one makes the change of variables $u=(\alpha-2) / 3$ and $v=(\beta-2) / 3$, one obtains the presentation in [2, Example 6.6]

$$
\mathbb{Q}_{3}[u, v] /\left(\alpha^{2}-\alpha-2, \beta^{2}-\beta-2, \alpha \beta-2(\alpha+\beta-2)\right) .
$$

Recall that after complexification we can determine the multiplicative structure in general (see Theorem 0.2). 
There are interesting discussions about Euler characteristics and maps of groups inducing isomorphisms on homology in [1] and [2] which also apply to our setting.

Remark 7.6 (Hodgkin's computation). Let $\Gamma^{n}$ be the mapping class group of the sphere $S^{2}$ with $n$ punctures for $n \geq 3$. Hodgkin computes rationally the $K$-theory of $B \Gamma^{n}$ with coefficients in the $p$-adic integers $\mathbb{Z}_{p}$ using Adem's formula in [1]. The main work done in the paper by Hodgkin [14, Proposition 2.2 and Theorem 2] is to figure out the set of conjugacy classes of elements of order $p^{s}$ for each prime $p$ and integer $s \geq 1$ and the rank of $K^{k}\left(B C_{G}\langle g\rangle\right) \otimes_{\mathbb{Z}}$ $\mathbb{Q} \cong \prod_{i \in \mathbb{Z}} H^{2 i+k}\left(B C_{G}\langle g\rangle ; \mathbb{Q}\right)$ for each element $g \in \Gamma^{n}$ of prime power order. One can identify $K^{*}\left(B C_{G}\langle g\rangle\right) \otimes_{\mathbb{Z}} \mathbb{Q}$ with $\left(K^{*}\left(B K^{r}\right) \otimes_{\mathbb{Z}} \mathbb{Q}\right)^{\Sigma_{r}}$ or with $\left(K^{*}\left(B K^{r}\right) \otimes_{\mathbb{Z}} \mathbb{Q}\right)^{\Sigma_{r-2} \times \Sigma_{2}}$ for appropriate integers $r$ depending only on the order of $g$, where $K^{r}$ is the pure mapping class group of $S^{2}$ with $r$ punctures and $\Sigma_{t}$ denotes the symmetric group of permutation of the set consisting of $t$ elements. Thus one obtains with Theorem 0.1 the precise structure of the $\mathbb{Q}$-vector spaces $K^{k}\left(B \Gamma^{n}\right) \otimes_{\mathbb{Z}} \mathbb{Q}$. It may be worthwhile to investigate the product structure on the cohomology $H^{*}\left(C_{G}\langle g\rangle ; \mathbb{C}\right)$ since this would lead to a computation of $K^{*}\left(B \Gamma^{n}\right) \otimes_{\mathbb{Z}} \mathbb{C}$ including its multiplicative structure by Theorem 0.2 .

Remark 7.7 (Criterion for torsionfree). Let $G$ be a discrete group with a finite model for $\underline{E} G$. Then the following assertions are equivalent:

(a) $G$ is torsionfree;

(b) The abelian group $K^{k}(B G)$ is finitely generated for $k \in \mathbb{Z}$;

(c) The rational vector space $K^{k}(B G) \otimes_{\mathbb{Z}} \mathbb{Q}$ is finite dimensional for $k \in \mathbb{Z}$.

An application of the Atiyah-Hirzebruch spectral sequence proves the implication $(\mathrm{a}) \Rightarrow(\mathrm{b})$. The implication (b) $\Rightarrow(\mathrm{c})$ is obvious. The implication (c) $\Rightarrow(\mathrm{a})$ follows from Theorem 0.1 since $\mathbb{Q}_{p}$ is an infinite dimensional $\mathbb{Q}$-vector space.

Remark 7.8 (Torsion prime to the order of finite subgroups). Suppose that there is a finite model for $\underline{E} G$. Let the ring $\Lambda^{G}$ be the subring of $\mathbb{Q}$ obtained by inverting the orders of finite subgroups of $G$. We state without giving the details of the proof but referring to [16] that one can improve Theorem 0.1 to the statement that there is a $\Lambda^{G}$-isomorphism

$$
\begin{aligned}
\overline{\operatorname{ch}}_{G, \lambda}^{n}: K^{n}(B G) \otimes_{\mathbb{Z}} \Lambda^{G} \stackrel{\cong}{\rightrightarrows} \\
K^{n}(G \backslash \underline{E} G) \otimes_{\mathbb{Z}} \Lambda^{G} \times \prod_{p \text { prime }} \prod_{(g) \in \operatorname{con}_{p}(G)}\left(\prod_{i \in \mathbb{Z}} H^{2 i+n}\left(B C_{G}\langle g\rangle ; \mathbb{Q}_{p}\right)\right) .
\end{aligned}
$$

Consider a prime $q$ for which there exists no element order $q^{s}$ for some $s \geq 1$ in $G$, in other words, $q$ is not invertible in $\Lambda^{G}$. Then the projection $B G=$ $E G \times_{G} \underline{E} G \rightarrow G \backslash \underline{E} G$ induces an isomorphism

$$
\operatorname{tors}_{q}\left(K^{n}(G \backslash \underline{E} G)\right) \stackrel{\cong}{\rightarrow} \operatorname{tors}_{q}\left(K^{n}(B G)\right),
$$


if for an abelian group $A$ we denote by $\operatorname{tors}_{q}(A)$ the subgroup of elements $a \in A$ which are annihilated by some power of $q$. In particular $K^{n}(B G)$ contains $q$-torsion if and only if $K^{n}(G \backslash \underline{E} G)$ contains $q$-torsion. It can occur that $K^{n}(G \backslash \underline{E} G)$ contains elements of order $q^{s}$ for some $s \geq 1$ (see [17].) We will explain in [16] that the subgroup of torsion elements in $K^{n}(B G)$ is finite.

\section{References}

[1] A. Adem. On the $K$-theory of the classifying space of a group. Math. Annalen, 293:319-327, 1992.

[2] A. Adem. Characters and $K$-theory of discrete groups. Invent. Math., 114(3):489-514, 1993.

[3] M. Artin and B. Mazur. Etale homotopy. Lecture Notes in Mathematics, No. 100. Springer-Verlag, Berlin, 1969.

[4] M. F. Atiyah. Characters and cohomology of finite groups. Inst. Hautes Études Sci. Publ. Math., (9):23-64, 1961.

[5] M. F. Atiyah and I. G. Macdonald. Introduction to commutative algebra. Addison-Wesley Publishing Co., Reading, Mass.-London-Don Mills, Ont., 1969.

[6] M. F. Atiyah and G. B. Segal. Equivariant $K$-theory and completion. $J$. Differential Geometry, 3:1-18, 1969.

[7] M. F. Atiyah and D. O. Tall. Group representations, $\lambda$-rings and the $J$ homomorphism. Topology, 8:253-297, 1969.

[8] P. Baum, A. Connes, and N. Higson. Classifying space for proper actions and $K$-theory of group $C^{*}$-algebras. In $C^{*}$-algebras: 1943-1993 (San Antonio, TX, 1993), pages 240-291. Amer. Math. Soc., Providence, RI, 1994.

[9] G. E. Bredon. Introduction to compact transformation groups. Academic Press, New York, 1972. Pure and Applied Mathematics, Vol. 46.

[10] K. S. Brown. Cohomology of groups, volume 87 of Graduate Texts in Mathematics. Springer-Verlag, New York, 1982.

[11] J. F. Davis and W. Lück. Spaces over a category and assembly maps in isomorphism conjectures in $K$ - and $L$-theory. $K$-Theory, 15(3):201-252, 1998.

[12] W. Dicks and M. J. Dunwoody. Groups acting on graphs. Cambridge University Press, Cambridge, 1989.

[13] M. J. Dunwoody. Accessibility and groups of cohomological dimension one. Proc. London Math. Soc. (3), 38(2):193-215, 1979. 
[14] L. Hodgkin. $K$-theory of mapping class groups: general $p$-adic $K$-theory for punctured spheres. Math. Z., 218(4):611-634, 1995.

[15] S. Jackowski and B. Oliver. Vector bundles over classifying spaces of compact Lie groups. Acta Math., 176(1):109-143, 1996.

[16] M. Joachim and W. Lück. Topological $K$-(co-)homology of classifying spaces of discrete groups. in preparation, 2005.

[17] I. J. Leary and B. E. A. Nucinkis. Every CW-complex is a classifying space for proper bundles. Topology, 40(3):539-550, 2001.

[18] I. J. Leary and B. E. A. Nucinkis. Some groups of type VF. Invent. Math., 151(1):135-165, 2003.

[19] W. Lück. The relation between the Baum-Connes conjecture and the trace conjecture. Invent. Math., 149(1):123-152, 2002.

[20] W. Lück. Equivariant cohomological Chern characters. Preprintreihe SFB 478 - Geometrische Strukturen in der Mathematik, Heft 309, Münster, arXiv:math.GT/0401047, to appear in International Journal of Algebra and Computation, 2004.

[21] W. Lück. Survey on classifying spaces for families of subgroups. Preprintreihe SFB 478 - Geometrische Strukturen in der Mathematik, Heft 308, Münster, arXiv:math.GT/0312378 v1, to appear in Progress in Mathematics, Birkhäuser, 2004.

[22] W. Lück, H. Reich, and M. Varisco. Commuting homotopy limits and smash products. K-Theory, 30(2):137-165, 2003. Special issue in honor of Hyman Bass on his seventieth birthday. Part II.

[23] W. Lück and R. Stamm. Computations of $K$ - and $L$-theory of cocompact planar groups. K-Theory, 21(3):249-292, 2000.

[24] R. C. Lyndon and P. E. Schupp. Combinatorial group theory. SpringerVerlag, Berlin, 1977. Ergebnisse der Mathematik und ihrer Grenzgebiete, Band 89.

[25] J.-P. Serre. Cohomologie des groupes discrets. In Prospects in mathematics (Proc. Sympos., Princeton Univ., Princeton, N.J., 1970), pages 77-169. Ann. of Math. Studies, No. 70. Princeton Univ. Press, Princeton, N.J., 1971.

[26] J.-P. Serre. Linear representations of finite groups. Springer-Verlag, New York, 1977. Translated from the second French edition by Leonard L. Scott, Graduate Texts in Mathematics, Vol. 42.

[27] J.-P. Serre. Trees. Springer-Verlag, Berlin, 1980. Translated from the French by J. Stillwell. 
[28] C. Soulé. The cohomology of $\mathrm{SL}_{3}(\mathbf{Z})$. Topology, 17(1):1-22, 1978.

[29] R. M. Switzer. Algebraic topology-homotopy and homology. SpringerVerlag, New York, 1975. Die Grundlehren der mathematischen Wissenschaften, Band 212.

[30] M. Tezuka and N. Yagita. Complex $K$-theory of $B \mathrm{sl}_{3}(\mathbf{z})$. K-Theory, 6(1):87-95, 1992. 